\title{
WestVirginiaUniversity
}

THE RESEARCH REPOSITORY @ WVU

Graduate Theses, Dissertations, and Problem Reports

2016

\section{Cooperative maneuver enabled UAV relative localization}

Jared Strader

Follow this and additional works at: https://researchrepository.wvu.edu/etd

\section{Recommended Citation}

Strader, Jared, "Cooperative maneuver enabled UAV relative localization" (2016). Graduate Theses, Dissertations, and Problem Reports. 6735.

https://researchrepository.wvu.edu/etd/6735

This Thesis is protected by copyright and/or related rights. It has been brought to you by the The Research Repository @ WVU with permission from the rights-holder(s). You are free to use this Thesis in any way that is permitted by the copyright and related rights legislation that applies to your use. For other uses you must obtain permission from the rights-holder(s) directly, unless additional rights are indicated by a Creative Commons license in the record and/ or on the work itself. This Thesis has been accepted for inclusion in WVU Graduate Theses, Dissertations, and Problem Reports collection by an authorized administrator of The Research Repository @ WVU. For more information, please contact researchrepository@mail.wvu.edu. 


\title{
COOPERATIVE MANEUVER ENABLED UAV RELATIVE LOCALIZATION
}

\author{
Jared Strader
}

\author{
Thesis submitted to the \\ Benjamin M. Statler College of Engineering and Mineral Resources \\ at West Virginia University \\ in partial fulfillment of the requirements for the degree of \\ Master of Science \\ in \\ Mechanical Engineering
}

Yu Gu, Ph.D., Chair

Jason Gross, Ph.D.

Marvin Cheng, Ph.D.

Department of Mechanical and Aerospace Engineering

\author{
Morgantown, West Virginia \\ 2016
}

Keywords: cooperative localization, range-only measurements, relative localization

Copyright (C) 2016 


\title{
ABSTRACT \\ Cooperative Maneuver Enabled UAV Relative Localization
}

\author{
Jared Strader
}

The ability to localize itself in an environment is essential for any autonomous vehicle. In applications involving multiple Unmanned Aerial Vehicles (UAVs) such as formation flight, surveillance, and mapping, the relative pose of each UAV provides vital information for multiple vehicles to coordinate effectively. The majority of cooperative localization tasks involving multiple UAVs assume the transformation between reference frames, but this information is not always provided in the absence of landmarks or a Global Positioning System (GPS).

This thesis presents a method for estimating the relative pose of a pair of UAVs using range-only measurements. In this method, there is no prior information assumed about the relative pose of each UAV. Instead, the trajectories are constrained in order to leverage the circumstances in which the range-only measurements are obtained during flight. To augment this limited information, motion is used to construct a graph of the UAV trajectories using only distance measurements. Using the constructed graph, four potential solutions are derived for the relative pose, and a batch estimate is performed to obtain numerical estimates of each of the four solutions. Using the batch estimate, a cooperative maneuver can be performed in order to obtain a unique solution. The sensitivity to the trajectory and measurement noise are analyzed through a Monte Carlo analysis. 


\section{Acknowledgments}

First and foremost, I would like to thank my committee chairman Dr. Yu Gu for his guidance, contribution, and support. I would also like to thank my wife Nancy for her love and encouragement throughout my graduate study. This work would not have been possible without her support. I would like to thank my committee members Dr. Jason Gross and Dr. Marvin Cheng for taking time to review and contribute to this research effort. Many thanks to Deborah Willis for helping with logistics. I would like to thank the members of the Interactive Robotics Laboratory that I have had the privilege to work with closely over the past couple years. This includes Kyle Lassak, Alex Hypes, Scott Harper, Tanmay Mandal, Matt Rhudy, and Caleb Rice. I would also like to thank Ryan Watson and Jeremy Hardy from the Navigation Lab at West Virginia University. I would like to thank all my friends that I have worked with during my time thus far at West Virginia University. And last, but certainly not least, I would like to thanks my brothers, Isaac and Noah, and my sisters, Sarah and Rachel, for their encouragement and support throughout my life. 


\section{Contents}

$\begin{array}{ll}\text { Abstract } & \text { ii }\end{array}$

Acknowledgments $\quad$ iii

List of Figures $\quad$ vi

1 Introduction 1

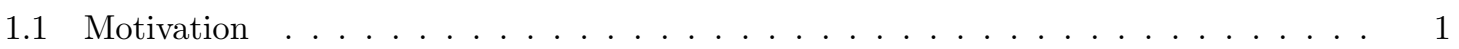

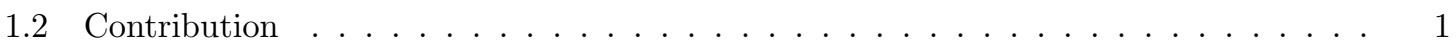

1.3 Overview of Thesis $\ldots \ldots \ldots \ldots \ldots \ldots \ldots \ldots \ldots \ldots$

2 Background $\quad 3$

3 Problem Formulation $\quad 6$

3.1 Notation . . . . . . . . . . . . . . . . . . . . . . . . . 6

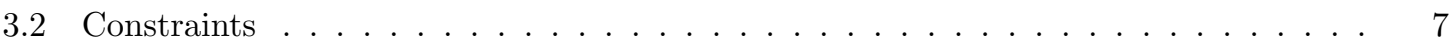

3.3 Relation to Mechanical Linkages . . . . . . . . . . . . . . . . . 8

4 Analytical Solutions with Three Measurements 11

4.1 Relative Heading . . . . . . . . . . . . . . . . . . . . . . . . . . . 11

4.2 Relative Bearing . . . . . . . . . . . . . . . . . . . . . . 15

$4.3 \quad$ Special Cases . . . . . . . . . . . . . . . . . . . . . . . . 16

$4.3 .1 \quad$ Same Line Trajectory . . . . . . . . . . . . . . . . . . . . . . 17

$4.3 .2 \quad$ Parallel with Equal Velocities . . . . . . . . . . . . . . . . . . 18

4.3 .3 Parallel with Different Velocities _. . . . . . . . . . . . . . . . . . . 19

4.3 .4 Intersecting Lines . . . . . . . . . . . . . . . . . . . . . . . . . . . . 19 
5 Finding a Unique Solution

5.1 Cooperative Maneuver . . . . . . . . . . . . . . . . . . . . . . . 21

5.1 .1 Assigning Weights to the Solutions . . . . . . . . . . . . . . . . . 22

5.1 .2 Constraints for the Cooperative Maneuver . . . . . . . . . . . . . . . . 24

6 Noisy Measurements 28

6.1 Estimating the Relative Pose . . . . . . . . . . . . . . . . . . . . . 28

6.2 Sensitivity Analysis . . . . . . . . . . . . . . . . . . . . . 31

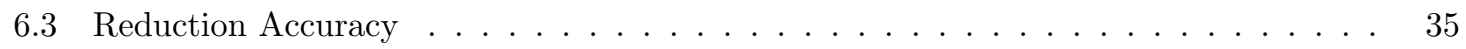

7 Concluding Remarks $\quad 38$

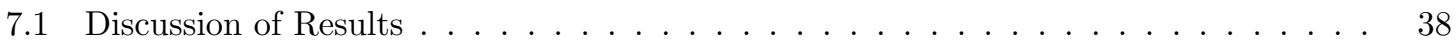

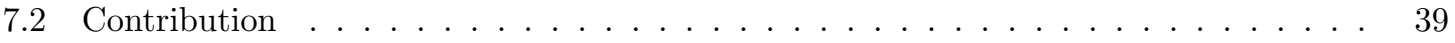

7.3 Future Work . . . . . . . . . . . . . . . . . . . . . . . . . 39 


\section{List of Figures}

3.1 Trajectories of platforms $A$ and $B$ after traveling through three locations. The position of each platform is denoted by $A_{1}, A_{2}, A_{3}$ for platform $A$ and $B_{1}, B_{2}, B_{3}$ for platform $B$. The displacement in distance between locations is denoted as $d_{A 1}, d_{A 2}$ for platform $A$ and $d_{B 1}, d_{B 2}$ for platform $B \ldots \ldots \ldots \ldots \ldots \ldots \ldots \ldots \ldots$

3.2 Single configuration of 5 bar linkage constructed from the UAV trajectories for 3 locations where $r_{1}=d_{A 1}+d_{A 2}, r_{2}=d_{3}, r_{3}=d_{B 1}+d_{B 2}, r_{4}=d_{1}$, and $r_{5}=d_{2}$. Note $\theta_{1}$ is not displayed because $\theta_{1}=0 \ldots \ldots \ldots \ldots \ldots \ldots \ldots \ldots \ldots \ldots \ldots \ldots$

4.1 Illustration of loop closure equation presented in Equation $(4.1) \ldots \ldots \ldots \ldots \ldots$

4.2 Illustration of loop closure equation presented in Equation (4.2) . . . . . . . . . . 12

4.3 Illustration of loop closure equation presented in Equation $(4.19) \ldots \ldots \ldots$

4.4 Configuration of platform $A$ and platform $B$ if both platforms continue on the current trajectory to a fourth location. . . . . . . . . . . . . . . . . . .

4.5 Path of platform $A$ and platform $B$ when the platforms are traveling towards each

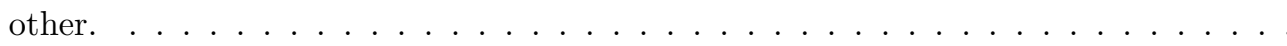

4.6 Path of platform $A$ and platform $B$ the platforms are traveling parallel with equal velocities. In this case, $\theta_{3}=0, d_{A 1}=d_{A 2}=d_{B 1}=d_{B 2}$, and $d_{1}=d_{2}=d_{3}$, so in (4.17) and (4.25), $r_{2}=r_{4}=r_{5}$ and $r_{1}=r_{2} \ldots \ldots \ldots \ldots \ldots \ldots \ldots \ldots \ldots \ldots \ldots \ldots$

4.7 Path of platform $A$ and platform $B$ when platforms are traveling parallel with different velocities. In this case, $\theta_{3}=0, d_{A 1}+d_{A 2} \neq d_{B 1}+d_{B 2} \ldots \ldots \ldots \ldots$

4.8 Path of platform $A$ and platform $B$ when platforms are traveling along intersecting lines. In this case, $\theta_{3} \neq 0$, and there are two solutions for the relative orientation and four solutions for the relative position. The solutions for the relative position of platform $B$ are given by $B_{i, j}$ where $i$ is the location number and $j$ is the solution number. 
5.1 Illustration of propagating the position of platform $B$ to a fourth location if platform $B$ remains on the current trajectory and platform $A$ changes trajectories, so that the fourth location is on the same line as the previous trajectory. Red indicates the true solutions, and blue indicates the false solutions. Note that in this case $\operatorname{dist}\left(B_{4,1}, A_{4}\right)=\operatorname{dist}\left(B_{4,3}, A_{4}\right)$ and $\operatorname{dist}\left(B_{4,2}, A_{4}\right)=\operatorname{dist}\left(B_{4,4}, A_{4}\right)$ are equivalent. . .

5.2 Illustration of propagating the position of platform $B$ to a fourth location if the platform $B$ remains on the current trajectory and platform $A$ changes trajectories, so that the fourth location does not like on the same line as the previous trajectory. Red indicates the true solutions, and blue indicates the false solutions. Note that in this case $\operatorname{dist}\left(B_{4,1}, A_{4}\right) \neq \operatorname{dist}\left(B_{4,2}, A_{4}\right) \neq \operatorname{dist}\left(B_{4,3}, A_{4}\right) \neq \operatorname{dist}\left(B_{4,4}, A_{4}\right)$. In addition, $\operatorname{dist}\left(B_{4,3}, A_{4}\right)$ and $\operatorname{dist}\left(B_{4,4}, A_{4}\right)$ are both greater than $\operatorname{dist}\left(B_{4,1}, A_{4}\right)$ and $\operatorname{dist}\left(B_{4,2}, A_{4}\right) 26$

5.3 Perpendicular bisector of the line $P Q$. The locus of all points equidistant from $P$ and $Q$ are contained on the perpendicular bisector of $P Q$. In the figures, the fourth location of $A$ is along the same trajectory of the first three locations. . . . . . . .

6.1 Average RMSE for the relative heading and relative bearing of platform $B$. The horizontal axis is the distance interval, and the vertical axis is the average RMSE error in degrees. 100 trials were completed for each distance interval. . . . . . . . . .

6.2 $\mathrm{CDF}$ of the relative heading and relative bearing of platform $B$ with respect to platform $A$ for various distance intervals. 100 trials were completed for each distance

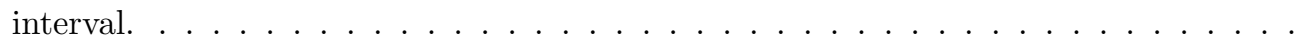

6.3 Average RMSE for the relative heading and relative bearing of platform $B$. The horizontal axis is the relative heading of platform $B$, and the vertical axis is the average RMSE error in degrees. 100 trials were completed for each relative heading of platform $B . \ldots \ldots \ldots \ldots \ldots \ldots \ldots \ldots \ldots \ldots \ldots \ldots \ldots \ldots \ldots \ldots$

6.4 CDF of the relative heading and relative bearing of platform $B$ with respect to platform $A$ for various relative headings of platform B. 100 trials were completed for each

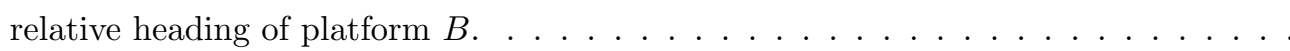

6.5 Average RMSE for the relative heading and relative bearing of platform $B$. The horizontal axis is the standard deviation of ranging measurements between platforms, and the vertical axis is the average RMSE error in degrees. 100 trials were completed for each level of ranging error. . . . . . . . . . . . . . . . . . 
6.6 $\mathrm{CDF}$ of the relative heading and relative bearing of platform $B$ with respect to platform $A$ for various levels of error for the ranging measurements between platforms. 100 trials were completed for each level of ranging error. . . . . . . . . . . . .

6.7 Average RMSE for the relative heading and relative bearing of platform $B$. The horizontal axis is the standard deviation as a fraction of the total displacement between locations, and the vertical axis is the average RMSE error in degrees. 100 trials were completed for each level of displacement error. Note the error is shown as the percentage of the displacement. . . . . . . . . . . . . .

6.8 $\mathrm{CDF}$ of the relative heading and relative bearing of platform $B$ with respect to platform $A$ for various levels of error for the displacement in position between locations. 100 trials were completed for each level of displacement error. Note the standard deviation of the displacement are shown as a percent of the total displacement. . . .

6.9 Percentage of trials that successfully predicted the correct solution for various levels of error for the ranging error. The horizontal axis is the heading of platform $B$ and the vertical axis is the percentage of trials that successfully predicted the correct solution. 36

6.10 Percentage of trials that successfully predicted the correct solution for various levels of error for the displacement error. The horizontal axis is the heading of platform $B$ and the vertical axis is the percentage of trials that successfully predicted the correct

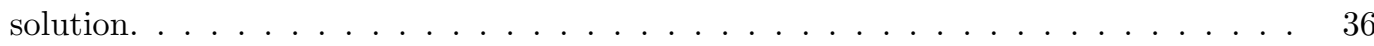




\section{Chapter 1}

\section{Introduction}

\subsection{Motivation}

In applications involving multiple UAVs such as formation flight, surveillance, cooperative control, and mapping, the relative pose between UAVs provides vital information for coordinating them effectively. In formation flight, the follower is controlled based on the relative position of the leader. For cooperative localization and mapping, the relative transformation between each UAVs reference frame is necessary to fuse information to construct a map of the environment. In the case that GPS is not available, each UAV must rely on relative sensor measurements in order to navigate in a common frame of reference. The majority of distributed estimation tasks require the transformation between reference frames in order to combine information.

\subsection{Contribution}

The most important contribution of this research is the enabling of relative localization of a pair of UAVs using coordinated motion. This work aims to obtains the relative pose between UAVs using range-only measurements by leveraging the ability to control the circumstances in which the measurements are obtained.

\subsection{Overview of Thesis}

The thesis is organized as follows: Chapter 2 presents an overview of the related work in cooperative localization and range-only navigation. Chapter 3 presents the relative pose problem and inspiration 
for the solution. Chapter 4 presents the derivation for the relative pose and shows that there are at most four solutions given three measurements. Chapter 5 describes the procedure and constraints necessary for obtaining a single unique solution through a cooperative maneuver. Chapter 6 discusses analyzes the sensitivity under various levels of noise. Finally, chapter 7 provides the concluding remarks and brief discussion on future research directions. 


\section{Chapter 2}

\section{Background}

The ability to localize itself in an environment is essential for any autonomous vehicle. If a robot or aircraft is localizing individually, the pose is determined using a combination of proprioceptive sensors and exteroceptive sensors. Proprioceptive sensors are sensors that monitor internal status including sensors such as wheel encoders, accelerometers, and gyroscopes. Exteroceptive sensors are sensors that obtain measurements from external sources including sensors such as ranging sensors, vision sensors, and contact sensors. If a group of robots or aircraft are able to share relative information such as range and bearing, then they are potentially able to localize cooperatively. To do this, the group can combine the relative information and obtain a more accurate estimate of the pose of the entire group [7]. This process is called cooperative localization, and it is an important for group members to coordinate effectively. The primary goal of cooperative localization is to increase the localization accuracy of the group by sharing sensory information [12]. The majority of work on cooperative localization fall in two major categories: the localization of multiple robot systems (ground, aerial, and underwater) and the localization of wireless sensor networks. Sensor localization will not be discussed since the focus of this thesis is cooperative maneuver enabled localization. A detailed survey on sensor localization can be found in [6].

In general, relative localization considers the scenario when a robot localizes using on board sensors. Absolute localization considers the scenario when a robot localizes to landmarks [4], beacons [5], or satellite signals such as GPS. Often cooperative localization is a combination of relative and absolute navigation. An early method for cooperative localization involved using subset of a group of robots as stationary landmarks while the remaining robots in the group move. [8, 9, 10, 11]. Later, similar methods were developed for localization using coordinated motion [13, 14, 15]. Meth- 
ods involving arbitrary motion were developed using different techniques. In [17], an approach is presented which combines information from a heterogeneous collection of robots through a single Kalman Filter but relies on external landmarks instead of the relative position of the group. In contrast, [16] presents an approach without the use of external information by using a combination of Maximum Likelihood Estimation and numerical optimization to estimate the pose of the group. Often these approaches are computationally expensive due to batch estimators, so methods using a recursive filter were developed in $[18,19]$. In addition, many researchers have made an effort to reduce the computational complexity by developing decentralized approaches. In [21], a distributed Maximum A Posteriori Estimation estimator is presented using the Distributed Conjugate Gradient algorithm to reduce the cost of computing the MAP estimates, but DCG algorithm requires synchronous communication, so this may not be possible depending on communication constraints. In [20], a single Kalman filter is distributed across multiple robots by sharing relative motion with the group. Several decoupled approaches exist in order to reduce computation by each sharing only the pose of nearby robots [22, 23]. Additionally, in [24] an Interlaced Extended Kalman Filter [25] is implemented by updating the relative position of pairs of nearby robots rather than updating the pose of the entire group. This exchange is limited to the pose and associated covariance in order to maintain a decoupled solution. In [26], a series of extended Kalman Filters are hierarchically distributed in a group of robots where the group is decomposed into subgroups, and for each subgroup, an extended Kalman Filter is used to estimate the configuration of the subgroup.

The majority of cooperative localization problems assume the transformation between reference frames, but in reality, this information is not always provided, especially in the absence of landmarks or GPS. In these cases, an initialization process is required to find the initial transformation between reference frames. In [1], a navigation filter is developed to enable target hand-off in a GPS-denied environment, but in order to run the filter, the initial transformation between reference frames is required. In many cases, aircraft may rely on sensors that only provide ranging information due to environmental or hardware constraints. In these cases, the initialization process is depending on relative motion. The majority of work on localization either require stationary landmarks or are not limited to range measurements. Localization using range-only measurements has been studied extensively for static sensor networks [6]. Several algorithms have been proposed for determining the relative position of each node; however, wireless sensor networks consist of many stationary nodes, and the relative orientation is not of interest in these algorithms. The problem of initialization and cooperative localization limited to range-only measurements have been investigated by multiple researchers $[27,28,29,30,32]$. The problem presented in this work is similar to the problems 
presented in $[27,28,29]$, but in contrast, this work presents a method for determining the relative position and orientation using coordinated motion rather than arbitrary motion. Specifically, it is shown that when two UAVs travel at constant velocity there are at most four potential solutions for the relative pose after collecting three range measurements. In addition, the total number of solutions are reduced to obtain a single unique solution by performing a single maneuver with one additional measurement. For certain trajectories, the relative pose may have less than three solutions after three measurements or the solution may be indeterminate. To verify the algorithm, Monte Carlo simulations are completed using the proposed method and the sensitivity to geometry is analyzed for various levels of measurement noise. 


\section{Chapter 3}

\section{Problem Formulation}

In this chapter, I present a method for determining the relative pose between two moving aircraft without the presence of external references such as GPS or landmarks. Specifically, I discuss how to estimate the relative pose between two moving aircraft using cooperative motion and the aircraft-toaircraft distance measurements. In order to expand this method to swarms or flocks, this method can be applied pairwise to all aircraft in a group to obtain the relative pose of all aircraft with respect to a common reference frame. The relative pose between aircraft is mandatory for cooperative localization and distributed tasks. Part of the work in this chapter has been published in conference papers. $[2,1]$ The first section will present the notation that will be used for the presenting the problem. The second section will present the constant velocity and constant altitude constraints. The third section discusses the relation to mechanical linkages and the analogous problem description.

\section{$3.1 \quad$ Notation}

In this chapter, two aircraft are considered, which are denoted as platform $A$ and platform $B$. Initially, each platform will move through a sequence of positions denoted as $\left\{A_{1}, A_{2}, \ldots, A_{n}\right\}$ for platform $A$ and $\left\{B_{1}, B_{2}, \ldots, B_{n}\right\}$ for platform $B$. At each location, both platforms measure the aircraft-to-aircraft range denoted as $\left\{d_{1}, d_{2}, \ldots, d_{n}\right\}$. In addition, the distance traveled between locations by each platform will be denoted as $\left\{d_{A 1}, d_{A 2}, d_{A(n-1)}\right\}$ for platform $A$ and $\left\{d_{B, 1}, d_{B, 2}, d_{B, n-1}\right\}$ for platform $B$ where $n$ is the total number of locations. The trajectory for platform $A$ and platform $B$ after traveling through three locations is presented in Figure 3.1. 


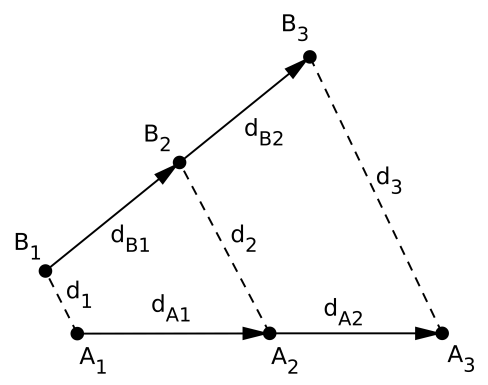

Figure 3.1: Trajectories of platforms $A$ and $B$ after traveling through three locations. The position of each platform is denoted by $A_{1}, A_{2}, A_{3}$ for platform $A$ and $B_{1}, B_{2}, B_{3}$ for platform $B$. The displacement in distance between locations is denoted as $d_{A 1}, d_{A 2}$ for platform $A$ and $d_{B 1}, d_{B 2}$ for platform $B$

\subsection{Constraints}

In order to derive a problem analogous to mechanical linkages, the aircraft must travel according to a few constraints. First, both platforms are assumed to navigate under the following assumptions:

- Each platform maintains a constant velocity.

- The range measurements between platforms at each time step are available to both platforms.

- The altitudes of each platform are available to both platforms.

- The displacement between observations for each platform are available to both platforms.

- GPS and common landmarks are not available to either of the platforms.

Using the altitude, the position of both platforms can be projected onto the $x y$ plane. Then, the problem can be solved for the relative position of the projection, and the solution can be back projected to obtain the three-dimensional solution. Second, both platforms must fly at a constant velocity. The velocity of the two platforms are not required to be equivalent. The constant velocity assumption is required in order to form a rigid linkage and reduce the number of solutions for the relative position. Note that the altitude of each platform, the aircraft-to-aircraft range, and the distance traveled between locations are assumed to be known by both platforms, which can be provided through each UAV's inertial navigation system and a ranging radio link between the two vehicles. The initial relative position and orientation between platform is unknown by both platforms. 


\subsection{Relation to Mechanical Linkages}

Before deriving the solution, mechanical linkages and the relationship to the presented problem will briefly be discussed. The analogy is not required in order to derive the solutions, but the analogy inspired the derived solution and provides intuition. A linkage, or kinematic chain, is a mechanical system assembled from rigid bodies connected in order to control movement or transfer force. Links are rigid bodies containing at least two nodes, which are points for attachment to other links. Joints are the connections between at least two links, which allow motion between the connected links. The joints are modeled as providing ideal movement, which means the joints provide either pure rotation or pure translation. In this section, only pin joints, binary links, and ternary links are relevant for the presented problem. Binary links are links consisting of two nodes, and ternary links are links consisting of three nodes. Pin joints allow pure rotation between connected links.

The degrees of freedom of a rigid body are the number of distinct direction it is able to move in space. In the case of a linkage, the number of degrees of freedom is the number of parameters required in order to determine the configuration. For example, an unrestrained rigid body, such as a link in place, has three degrees of freedom because the link can rotate in one direction and translate in two directions. A kinematic pair is a connection between two rigid bodies that imposes constraints on the relative movement. Typically, linkages are used to control movement or transfer force, so linkages are designed in order provide a specific path or force for the output linkage. In order to understand the output of a certain linkage, linkage analysis can be performed in order to determine the position and angle of each link. In order to solve the relative localization problem, the UAV trajectories will be modeled as a linkage, and the resulting linkage will be analyzed in order to obtain the relative pose between UAVs.

Due to the constant velocity and constant altitude constraints, the arrangement of measurements is analogous to a linkage; therefore, the projection of the trajectories of each platform can be modeled as rigid linkage connected at the platform locations. At constant velocity, the trajectories of each platform can be modeled as rigid links with length equal to the displacement in position between locations. The links are denoted by $\left\{d_{A 1}, d_{A 2}, d_{A(n-1)}\right\}$ for platform $A$ and $\left\{d_{B 1}, d_{B 2}, d_{B(n-1)}\right\}$ for platform $B$. In addition, the range between platforms at each location can be modeled as rigid links. These links are represented by the range measurements and denoted by $\left\{d_{1}, d_{2}, \ldots, d_{n}\right\}$. The connection of the links can be modeled as pin joints placed at the locations of each platform. The number of joints and links in this linkage can be determined by the number of locations throughout the trajectory. For any number of locations, the length of the links are known from the displacement 
in position between locations and the aircraft-to-aircraft range measurements. The displacement in position between locations is provided by the on board navigation system of each platform, and the aircraft-to-aircraft range measurements can be provided using any ranging device of adequate performance. The platforms are assumed to be able to communicate the aircraft-to-aircraft range measurements and the displacement in position for each platform, so the length of all the links are known by both platforms for any number of locations.

The goal is to solve for the orientation of the each link with respect to the fixed link since this will provide the relative pose between platforms at each location. To determine the orientation of each link without prior information, the linkage must be a rigid structure since this would imply that there is only one solution within the neighborhood of the correct solution. Using the Kutzbach criterion, the mobility, $M$, can be obtained for any number of measurements given the number of links $n$, the number of single degree of freedom joints $j_{1}$, and the number of two degree of freedom joints, $j_{2}$. The Kutzbach criterion as defined in [33] is

$$
M=3(n-1)-2 j_{1}-j_{2} .
$$

If each platform travels through two locations and obtains a measurement at each location, the linkage will consist of four pin joints and four links in total to form a four bar mechanism. Considering the Kutzbach criterion for this four bar mechanism, this linkage consists of a single degree of freedom. This means that the configuration of the linkage is defined by a single parameter in addition to the link lengths [33], and the linkage is indeterminate without the prior knowledge of the orientation of one of the free links. If each platform travels through three locations and obtains a measurement at each location, the linkage will consist of six pin joints and seven links in total to form a seven bar mechanism. Since the platforms are traveling at constant velocities, the links representing the displacement can be combined to form a single rigid link for each platform. This means the number of links in the linkage is reduced from seven links to five links, so the linkage will consist of six pin joints and five links in total to form a five bar linkage. Considering the Kutzbach criterion for this five bar mechanism, this linkage consists of zero degrees of freedom. This means the linkage is a rigid structure, so prior information about the orientation of the links is not needed to solve for the configuration given the length of each link. This is roughly equivalent to adding a single rigid link between the fixed link and coupler in a four bar mechanism.

Using this analogy, the relative position of $B$ with respect to $A$ can be obtained by solving the loop closure equations of the five bar linkage formed from the displacement in position, distance 
between platforms, and the locations of platform $A$ and platform $B$. Each link is defined as a vector defined by the link length $r_{1}=d_{A 1}+d_{A 2}, r_{2}=d_{3}, r_{3}=d_{B 1}+d_{B 2}, r_{4}=d_{1}$, and $r_{5}=d_{2}$, and the angle between the horizontal axis and the link $\theta_{1}, \theta_{2}, \theta_{3}, \theta_{4}$, and $\theta_{5}$. The linkage constructed from the trajectories of platform $A$ and platform $B$ is presented in Fig. 3.2. Since the goal is to obtain the relative position of platform $B$ with respect to platform $A$, the displacement in position of platform $A, r_{1}$, is set as the fixed link, so the angle of each link is relative to $r_{1}$. This means the linkage is constructed in the inertial frame of platform $A$. Note $\theta_{1}$ is not presented in Fig. 3.2 because the free links are relative to the fixed link, $r_{1}$.

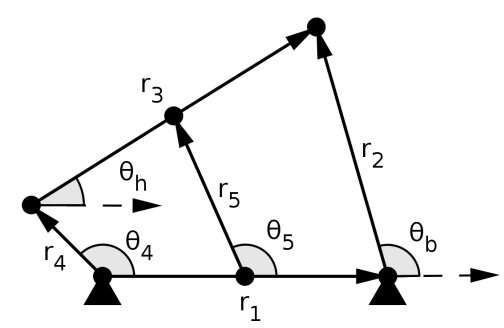

Figure 3.2: Single configuration of 5 bar linkage constructed from the UAV trajectories for 3 locations where $r_{1}=d_{A 1}+d_{A 2}, r_{2}=d_{3}, r_{3}=d_{B 1}+d_{B 2}, r_{4}=d_{1}$, and $r_{5}=d_{2}$. Note $\theta_{1}$ is not displayed because $\theta_{1}=0$. 


\section{Chapter 4}

\section{Analytical Solutions with Three Measurements}

In this chapter, the relative pose between the projections of each platform will be derived for three aircraft-to-aircraft range measurements using the linkage analogy provided in the previous chapter. Note that the relative pose is derived in polar coordinates, so the relative pose of each platform is defined by the heading, bearing, and distance as seen in Figure 3.2. Note that in this section the relative heading will be referred to as $\theta_{h}$ and the relative bearing will be referred to as $\theta_{b}$.

\subsection{Relative Heading}

The first loop closure equation for the linkage presented in Figure 3.2 in vector form is defined by Equation (4.1).

$$
\overrightarrow{r_{1}}+\overrightarrow{r_{2}}=\frac{d_{A 1}}{r_{1}} \overrightarrow{r_{1}}+\frac{d_{B 2}}{r_{3}} \overrightarrow{r_{3}}+\overrightarrow{r_{5}}
$$

An illustration of loop defined by Equation (4.1) is provided in Figure 4.1. The second loop closure equation for the linkage presented in Figure 3.2 in vector form is defined by Equation (4.2).

$$
\frac{d_{A 1}}{r_{1}} \overrightarrow{r_{1}}+\overrightarrow{r_{5}}=\frac{d_{B 1}}{r_{3}} \overrightarrow{r_{3}}+\overrightarrow{r_{4}}
$$

An illustration of loop defined by Equation (4.2) is provided in Figure 4.2. Equation (4.1) and Equation (4.2) can be rearranged and separated into $x$ and $y$ components as presented in Equation 

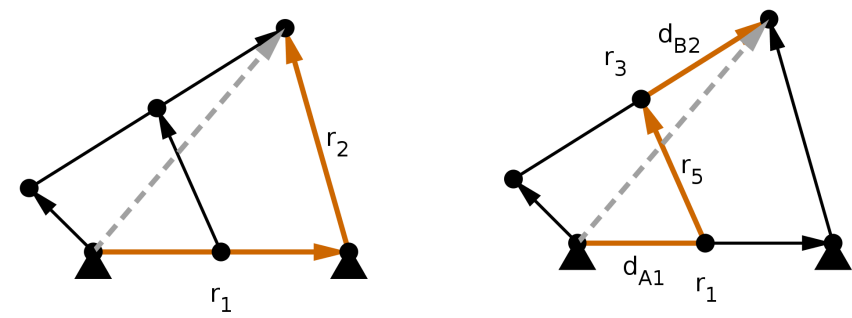

Figure 4.1: Illustration of loop closure equation presented in Equation (4.1)
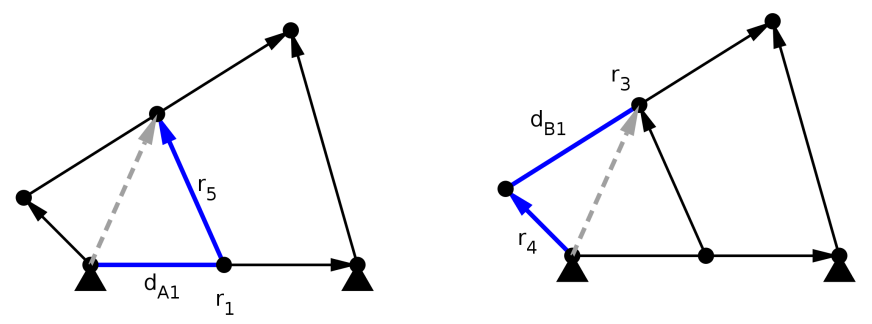

Figure 4.2: Illustration of loop closure equation presented in Equation (4.2).

(4.3) and Equation (4.4). Note that $r_{1}-d_{A 1}=d_{A 2}$ and $r_{3}-d_{B 1}=d_{B 2}$.

$$
\begin{aligned}
& x: r_{2} \sin \theta_{b}=r_{5} \sin \theta_{5}+d_{B 2} \sin \theta_{h}+d_{A 2} \sin \theta_{1} \\
& y: r_{2} \cos \theta_{b}=r_{5} \cos \theta_{5}+d_{B 2} \cos \theta_{h}+d_{A 2} \cos \theta_{1} \\
& x: r_{4} \sin \theta_{4}=r_{5} \sin \theta_{5}-d_{B 1} \sin \theta_{h}+d_{A 1} \sin \theta_{1} \\
& y: r_{4} \cos \theta_{4}=r_{5} \cos \theta_{5}-d_{B 1} \cos \theta_{h}+d_{A 1} \cos \theta_{1}
\end{aligned}
$$

Since the links are relative to $r_{1}$, the angle of $r_{1}$ does not change the outcome of the solution. Let $\theta_{1}=\pi / 2$. The result is presented in Equation (4.5) and Equation (4.6).

$$
\begin{aligned}
& x: r_{2} \sin \theta_{b}=r_{5} \sin \theta_{5}+d_{B 2} \sin \theta_{h}+d_{A 2} \\
& y: r_{2} \cos \theta_{b}=r_{5} \cos \theta_{5}+d_{B 2} \cos \theta_{h}+d_{A 2} \\
& x: r_{4} \sin \theta_{4}=r_{5} \sin \theta_{5}-d_{B 1} \sin \theta_{h}+d_{A 1} \\
& y: r_{4} \cos \theta_{4}=r_{5} \cos \theta_{5}-d_{B 1} \cos \theta_{h}+d_{A 1}
\end{aligned}
$$


Now, the equations can be squared and summed as presented in Equation (4.7) and Equation (4.8).

$$
\begin{aligned}
& r_{2}^{2}\left(\sin ^{2} \theta_{b}+\cos ^{2} \theta_{b}\right)=\left(r_{5} \sin \theta_{5}+d_{B 2} \sin \theta_{h}-d_{A 2}\right)^{2}+\left(r_{5} \cos \theta_{5}+d_{B 2} \cos \theta_{h}\right)^{2} \\
& r_{4}^{2}\left(\sin ^{2} \theta_{4}+\cos ^{2} \theta_{4}\right)=\left(r_{5} \sin \theta_{5}-d_{B 1} \sin \theta_{h}+d_{A 1}\right)^{2}+\left(r_{5} \cos \theta_{5}-d_{B 1} \cos \theta_{h}\right)^{2}
\end{aligned}
$$

Using the the Pythagorean trigonometric identity given by $\sin \theta^{2}+\cos \theta^{2}=1, \theta_{b}$ can be eliminated as presented in Equation (4.9) and Equation (4.10).

$$
\begin{aligned}
& r_{2}^{2}=\left(r_{5} \sin \theta_{5}+d_{B 2} \sin \theta_{h}-d_{A 2}\right)^{2}+\left(r_{5} \cos \theta_{5}+d_{B 2} \cos \theta_{h}\right)^{2} \\
& r_{4}^{2}=\left(r_{5} \sin \theta_{5}-d_{B 1} \sin \theta_{h}+d_{A 1}\right)^{2}+\left(r_{5} \cos \theta_{5}-d_{B 1} \cos \theta_{h}\right)^{2}
\end{aligned}
$$

Now the equations can be expanded and terms can be canceled as presented in Equation (4.11) and Equation (4.12).

$$
\begin{aligned}
r_{2}^{2}= & r_{5}^{2} \sin ^{2} \theta_{5}+r_{5} d_{B 2} \sin \theta_{h} \sin \theta_{5}-d_{A 2} r_{5} \sin \theta_{5} \\
& +r_{5} d_{B 2} \sin \theta_{h} \sin \theta_{5}+d_{B 2}^{2} \sin ^{2} \theta_{h}-d_{A 2} d_{B 2} \sin \theta_{h} \\
& -d_{A 2} r_{5} \sin \theta_{5}-d_{A 2} d_{B 2} \sin \theta_{h}+d_{A 2}^{2} \\
& +r_{5}^{2} \cos ^{2} \theta_{5}+2 d_{B 2} r_{5} \cos \theta_{h} \cos \theta_{5}+d_{B 2}^{2} \cos ^{2} \theta_{h} \\
r_{4}^{2}= & r_{5}^{2} \sin ^{2} \theta_{5}-r_{5} d_{B 1} \sin \theta_{h} \sin \theta_{5}+d_{A 1} r_{5} \sin \theta_{5} \\
& -r_{5} d_{B 1} \sin \theta_{h} \sin \theta_{5}-d_{B 1}^{2} \sin ^{2} \theta_{h}-d_{A 1} d_{B 1} \sin \theta_{h} \\
& +d_{A 1} r_{5} \sin \theta_{5}-d_{A 1} d_{B 1} \sin \theta_{h}+d_{A 1}^{2} \\
& +r_{5}^{2} \cos ^{2} \theta_{5}-2 d_{B 1} r_{5} \cos \theta_{h} \cos \theta_{5}+d_{B 1}^{2} \cos ^{2} \theta_{h}
\end{aligned}
$$

Simplifying and combining terms, Equation (4.13) and Equation (4.14) are obtained.

$$
r_{2}^{2}=r_{5}^{2}+r_{5}\left(2 d_{B 2} \sin \theta_{h} \sin \theta_{5}+2 d_{B 2} \cos \theta_{h} \cos \theta_{5}-2 d_{A 2} \sin \theta_{5}\right)-2 d_{A 2} d_{B 2} \sin \theta_{h}+d_{A 2}^{2}+d_{B 2}^{2}
$$




$$
r_{4}^{2}=r_{5}^{2}-r_{5}\left(2 d_{B 1} \sin \theta_{h} \sin \theta_{5}+2 d_{B 1} \cos \theta_{h} \cos \theta_{5}-2 d_{A 1} \sin \theta_{5}\right)-2 d_{A 1} d_{B 1} \sin \theta_{h}+d_{A 1}^{2}+d_{B 1}^{2}
$$

If platform $A$ and platform $B$ are moving at a constant speed, $d_{A 1}=d_{A 2}=d_{A}$ and $d_{B 1}=d_{B 2}=d_{B}$. Substituting these values and combining (4.13) and (4.14), a single equation is obtained for $\theta_{h}$ as a function of the link lengths.

$$
r_{2}^{2}+r_{4}^{2}=2 r_{5}^{2}-4 d_{A} d_{B} \sin \theta_{h}+2 d_{A}^{2}+2 d_{B}^{2}
$$

Equation (4.15) can be rearranged to obtain a more simple solution. Let $r_{a}=-4 d_{A} d_{B}$ and $r_{b}=$ $r_{2}^{2}+r_{4}^{2}-2 r_{5}^{2}-2 d_{A}^{2}-2 d_{B}^{2}$. Substituting $r_{a}$ and $r_{b}$ in Equation (4.15), the following equations are obtained for the $\sin \theta_{h}$ and $\cos \theta_{h}$ when $\theta_{1}=\pi / 2$ and $\theta_{1}=0$, respectively.

$$
r_{a} \sin \theta_{h}=r_{b}
$$

$$
r_{a} \cos \theta_{h}=r_{b}
$$

The relative heading of platform $B$ with respect to platform $A$ has two solutions. Note that the angle between trajectories is not equal to the relative orientation. The relative orientation is equal in magnitude to the angle between trajectories, but the angle between trajectories is always positive when using the inverse cosine function, so the relative heading is best described by

$$
\theta_{h}= \pm \cos ^{-1} \frac{r_{b}}{r_{a}}
$$

The potential solutions for the relative bearing can be obtained using $\theta_{h}$, but it should be noted that there are two solutions for $\theta_{h}$; therefore, the resulting solutions for $\theta_{b}$ must be reflected across the path of the opposite platform. The procedure for estimating thet $a_{h}$ is presented in the following chapters, but the process is straightforward since there is a single solution for $\cos \theta_{h}$. This means that $\cos \theta_{h}$ can be estimated, then the result can be plugged into Equation (4.16) or Equation (4.17) to obtain $\theta_{h}$. 


\subsection{Relative Bearing}

Consider the following loop closure equation of the linkage presented in Fig. 3.2 defined by (4.19).

$$
\overrightarrow{r_{1}}+\overrightarrow{r_{2}}=\overrightarrow{r_{3}}+\overrightarrow{r_{4}}
$$

An illustration of loop defined by Equation (4.19) is provided in Figure 4.3. Similar to the relative
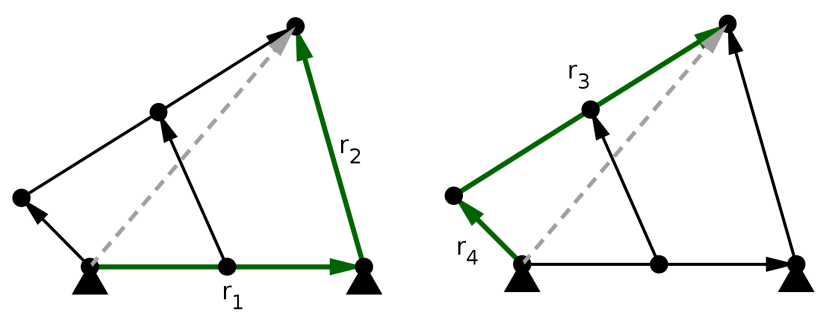

Figure 4.3: Illustration of loop closure equation presented in Equation (4.19)

heading, Equation (4.19) can be rearranged and separated into $x$ and $y$ components as presented in Equation (4.20).

$$
\begin{aligned}
& x: r_{4} \sin \theta_{4}=r_{1} \sin \theta_{1}+r_{2} \sin \theta_{b}-r_{3} \sin \theta_{h} \\
& y: r_{4} \cos \theta_{4}=r_{1} \cos \theta_{1}+r_{2} \cos \theta_{b}-r_{3} \cos \theta_{h}
\end{aligned}
$$

Since $\theta_{1}$ and the relative heading thet $a_{h}$ are given from the previous derivation, the constants $u$ and $v$ can be defined as

$$
\begin{aligned}
& u=r_{1} \sin \theta_{1}-r_{3} \sin \theta_{h} \\
& v=r_{1} \cos \theta_{1}-r_{3} \cos \theta_{h} .
\end{aligned}
$$

Substituting $u$ and $v$ into Equation (4.20), Equation (4.21) is obtained.

$$
\begin{aligned}
& x: r_{4} \sin \theta_{4}=r_{2} \sin \theta_{b}+u \\
& y: r_{4} \cos \theta_{4}=r_{2} \cos \theta_{b}+v
\end{aligned}
$$

Squaring and summing the $x$ and $y$ components of Equation (4.21), Equation (4.22) is obtained.

$$
r_{4}^{2}\left(\sin ^{2} \theta_{4}+\cos ^{2} \theta_{4}\right)=\left(u+r_{2} \sin \theta_{b}\right)^{2}+\left(v+r_{2} \cos \theta_{b}\right)^{2}
$$


Using the the Pythagorean trigonometric identity given by $\sin \theta^{2}+\cos \theta^{2}=1, \theta_{4}$ can be eliminated. Rearranging Equation (4.23) is obtained.

$$
r_{4}^{2}-r_{2}^{2}-u^{2}-v^{2}=2 u r_{2} \sin \theta_{b}-2 v r_{2} \cos \theta_{b}
$$

Equation (4.23) has the form $a \sin \theta_{b}+b \cos \theta_{b}=c$, where

$$
\begin{aligned}
& a=2 u r_{2} \\
& b=-2 v r_{2} \\
& c=-r_{2}^{2}+r_{4}^{2}-u^{2}-v^{2} .
\end{aligned}
$$

Using the half-angle formulas, Equation (4.23) can be written in terms of tangent instead of sine and cosine where $t=\tan \theta_{b} / 2, \sin \theta_{b}=2 t /\left(1+t^{2}\right)$, and $\cos \theta_{b}=\left(1-t^{2}\right) /\left(1+t^{2}\right)$. Substituting these values into Equation (4.23) and multiplying $c$ by one, Equation (4.24) is obtained.

$$
a \frac{2 t}{1+t^{2}}+b \frac{1-t^{2}}{1+t^{2}}=c \frac{1+t^{2}}{1+t^{2}}
$$

Rearranging and multiplying Equation (4.24) by $1+t^{2}$, Equation (4.25) is obtained where $\alpha=-b-c$, $\beta=2 a$, and $\gamma=b-c$.

$$
\alpha t^{2}+\beta t+\gamma=0
$$

The equations for $\theta_{4}$ and $\theta_{5}$ have the same form as Equation (4.25) but are not necessary for obtaining the relative pose between platforms. Note that Equation (4.25) is a second order polynomial and is a function of the relative heading. Since there are at most two solutions for the relative heading and the relative bearing is a function of the relative heading, there are at most four solutions for the relative bearing. Note that if a fourth measurement is provided by continuing to an addition location as presented in Figure 4.4, two solutions still exist for the relative heading and four solutions still exist for the relative bearing.

\subsection{Special Cases}

Depending on the geometry of the trajectories, the number of possible solutions and singularity of the relative bearing may vary. When the platforms are traveling at constant velocity, there are only 


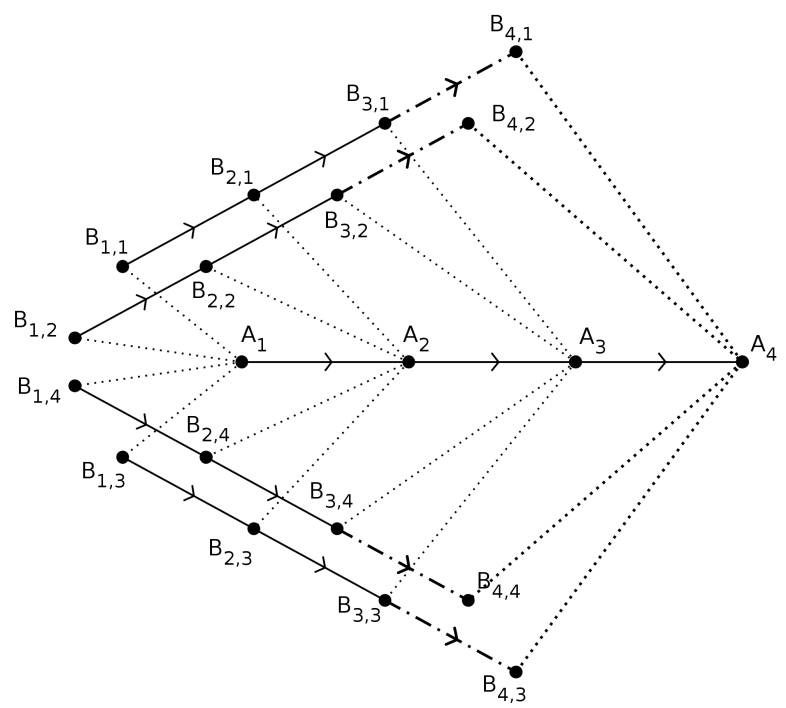

Figure 4.4: Configuration of platform $A$ and platform $B$ if both platforms continue on the current trajectory to a fourth location.

four possible cases for the geometry. The platforms must be either be traveling along the same line, traveling along parallel lines with equal velocity, traveling along parallel lines with different velocity, or traveling along intersecting lines. If the platforms are traveling along intersecting lines, there are at most four solutions for the relative position regardless of the velocity of each platform. For each case, the number of solutions for the relative position are different, but there are at most two solutions for the relative orientation in each case. Each case is easily detectable using the relationships between the displacement in position and the range between platforms. The solutions for each case are discussed in this section.

\subsubsection{Same Line Trajectory}

If the platforms are traveling along the same line in opposite directions there exists a single unique solution for the position and a single unique solution for the relative orientation. This is detectable by comparing the magnitude of the velocity vectors and the change in distance measurements between time steps. When the platforms are traveling in opposite directions along the same line, then

$$
\begin{aligned}
& d_{A 1}+d_{B 1}=\left|d_{1}-d_{2}\right| \\
& d_{A 2}+d_{B 2}=\left|d_{2}-d_{3}\right|
\end{aligned}
$$


Note that $d_{1}, d_{2}$, and $d_{3}$ are measurements of the distance between platforms and $d_{A}$ and $d_{B}$ are the displacement in position for platform $A$ and platform $B$, respectively. The absolute value is necessary because $d_{1}-d_{2}>0$ and $d_{2}-d_{3}>0$ when the platforms are traveling towards each other and $d_{1}-d_{2}<0$ and $d_{2}-d_{3}<0$ when the platforms are traveling away from each other. An illustration of this case is presented in Figure 4.5 for when the platforms are traveling along the same line towards each other.

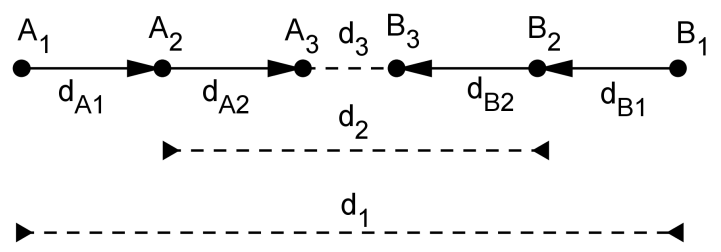

Figure 4.5: Path of platform $A$ and platform $B$ when the platforms are traveling towards each other.

If the platforms are traveling in the same direction, then $d_{1}-d_{2}=0$ and $d_{2}-d_{3}=0$, and there are an infinite number of solutions. This situation is discussed in the following section. In the case the platforms are not traveling along the same line, the change in the distance measurement between platforms will always be less than the sum of the distance traveled by each platform assuming there is no noise in the measurements.

\subsubsection{Parallel with Equal Velocities}

If the platforms are traveling parallel with equal velocities, there are infinite solutions for the relative position and a single unique solution for the relative orientation. An illustration of this case is presented in Figure 4.6.

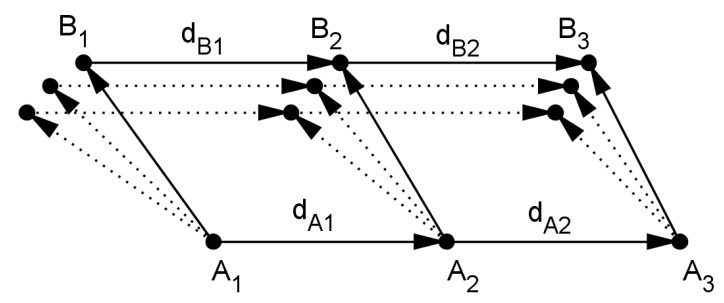

Figure 4.6: Path of platform $A$ and platform $B$ the platforms are traveling parallel with equal velocities. In this case, $\theta_{3}=0, d_{A 1}=d_{A 2}=d_{B 1}=d_{B 2}$, and $d_{1}=d_{2}=d_{3}$, so in (4.17) and (4.25), $r_{2}=r_{4}=r_{5}$ and $r_{1}=r_{2}$.

In this case, $d_{A 1}=d_{A 2}=d_{B 1}=d_{B 2}, r_{2}=r_{4}=r_{5}$ and $r_{1}=r_{3}$. If there values are substituted in (4.17), then $\cos \theta_{3}=1$. Then, $\theta_{1}=\theta_{h}=0$, so substituting these values in (4.25) causes the constants 
to equal zero. Therefore, $\theta_{b}$ is indeterminate because $(0) t_{2}^{2}+(0) t_{2}+(0)=0$ when the platforms are traveling parallel with equal velocities. This can easily be visualized from the constructed linkage. In this case, the linkage forms a parallelogram structure, so the link formed by the displacement in position of platform $B$ can rotate through an infinite number of positions.

\subsubsection{Parallel with Different Velocities}

If the platforms are traveling parallel at different speeds, there are two unique solutions for the relative position and a single unique solution for the relative orientation. In this case, $\theta_{1}=0$ and $\theta_{3}=0$ because the platforms are traveling parallel. By substituting $\theta_{1}=0$ and $\theta_{3}=0$ into (4.25), $\beta=4 r_{1} r_{2} \sin \theta_{1}-4 r_{2} r_{3} \sin \theta_{3}=0$, so $(4.25)$ becomes

$$
\alpha t^{2}+\gamma=0
$$

Note $\alpha \neq 0$ and $\gamma \neq 0$ if both platforms are in motion and are traveling at different velocities. Equation (4.28) has two solutions, which are equal in magnitude but have opposite signs. The solutions to (4.28) and the reflection are equivalent, so there are only two solutions for the relative position when the platforms are traveling parallel with different velocities. An illustration of this case is presented in Fig. 4.7 .

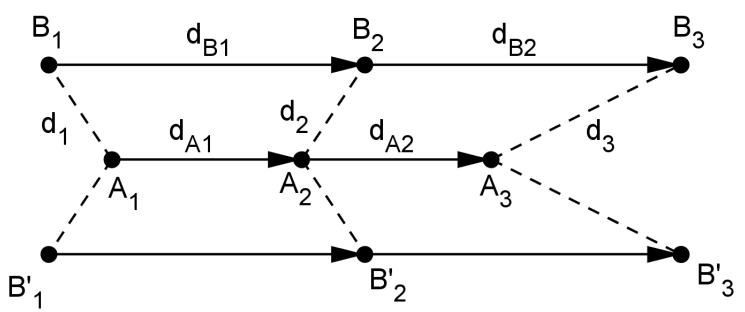

Figure 4.7: Path of platform $A$ and platform $B$ when platforms are traveling parallel with different velocities. In this case, $\theta_{3}=0, d_{A 1}+d_{A 2} \neq d_{B 1}+d_{B 2}$.

\subsubsection{Intersecting Lines}

If the platforms are not traveling parallel and are not traveling along the same line, there are four unique solutions for the relative position and two unique solutions for the relative orientation. In this case, $\theta_{3} \neq 0$, so there are two solutions for the relative orientation. Equation (4.25) has two solutions when the $\theta_{3} \neq 0$, and this solution may be reflected across the path of platform $A$ to account for the position and negative solution of the relative orientation. An illustration of this case 
is presented in Fig. 4.8. Notice the first and second solution are for the positive relative orientation. The solutions for the negative relative orientation are reflected across the path of platform $A$ to form the third and fourth solutions.

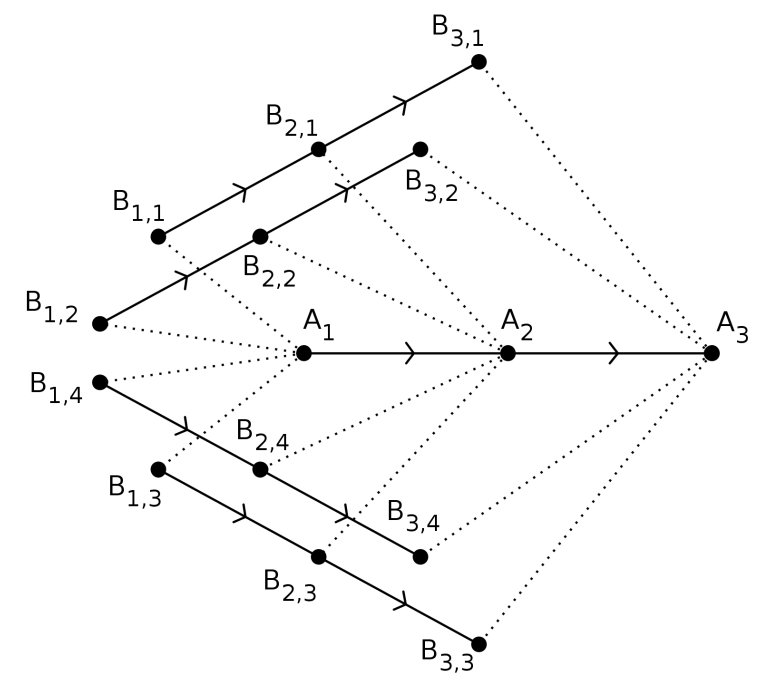

Figure 4.8: Path of platform $A$ and platform $B$ when platforms are traveling along intersecting lines. In this case, $\theta_{3} \neq 0$, and there are two solutions for the relative orientation and four solutions for the relative position. The solutions for the relative position of platform $B$ are given by $B_{i, j}$ where $i$ is the location number and $j$ is the solution number. 


\section{Chapter 5}

\section{Finding a Unique Solution}

In this section, the procedure for obtaining a unique solution will be discussed as well as the constraints and requirenments necessary in order to perform the cooperative maneuver. Given three measurements satisfying the constraints provided in the previous chapters, there are at most four solutions for the relative pose between platforms. There are two possible solutions for the relative heading each with equal magnitude but opposite signs, and there are two possible solutions for the relative bearing, which is a function of the relative heading. This means there are at most four solutions total for the relative pose given three measurements. If one of the platforms perform a maneuver and obtain at least one additional measurement, the total number of solutions can be reduced to a single unique solution. In rare cases, the system may be indeterminate, but this is easily detectable and will be discussed later in this chapter.

\subsection{Cooperative Maneuver}

The maneuver is described as cooperative because both platform must maintain a specific trajectory in order to find a unique solutions. After the platforms travel through each of the three locations, there are at most four possible solutions for the relative pose, which is a combination of the two possible solutions for the relative heading and the corresponding solutions for the relative bearing. To find the correct solution, a cooperative maneuver is used to assign weights to each of the four solutions. The case is considered where platform $A$ is the platform performing the maneuver, but the result is identical if platform $B$ is performing the maneuver. In this section, the procedure for finding a unique solution will be discussed as well as the constraints for performing the maneuver. 


\subsubsection{Assigning Weights to the Solutions}

Before examining the constraints of the cooperative of the maneuver, the method for assigning the weights to each of the solutions will be discussed. After performing the cooperative maneuver to reach the location at time $t_{k}$, the process to assign weights to the solutions is outlined as follows:

- Calculate the relative heading at time $t_{k}$ given measurements at time $t_{k-2}, t_{k-1}$, and $t_{k}$;

- Calculate the relative bearing at time $t_{k}$ given the relative heading and measurements at time $t_{k-2}, t_{k-1}$, and $t_{k}$

- For each of the four possible solutions, propagate the pose from time $t_{k}$ to time $t_{k+1}$;

- Calculate the distance between the current position at time $t_{k}$ and the propagated position at time $t_{k+1}$

- Calculate the difference between the calculated distance at time $t_{k+1}$ and the measured distance at time $t_{k+1}$

- Assign weights to each of the solutions as the inverse of the difference between the calculated distance and the measured distance;

- Keep the solution with the largest weight as the correct solution.

Note that the method for assigning weights to the solutions is based on the assumption that none of the four solutions after the maneuver will be equidistant from platform $A$. This requires platform $A$ to change trajectories if platform $A$ continues on the same trajectory all four solutions will be equidistant from platform $A$. The details of this assumption will be discussed later in this section. In the previous chapter, the analytical solution was presented for the relative heading and relative bearing, but the velocity was not discussed and is required for propagating the position of each of the four solutions. For simplicity, the position will be propagated in Cartesian coordinates rather than polar coordinates. The conversion between Cartesian and polar coordinates is straightforward and is provided in Equation (5.1).

$$
\begin{gathered}
x_{B}=d_{k} \cos \theta_{b} \\
y_{B}=d_{k} \sin \theta_{b}
\end{gathered}
$$

For each solution, the magnitude of the velocity can be obtained by dividing displacement in distance between locations by the change in time between locations. Since the displacement in distance 
between locations for platform $B$ is already known from the on board navigation system, the velocity for platform $B$ can be calculate by splitting the displacement into $x$ and $y$ components using the relative heading. The result is provided in Equation (5.2).

$$
\begin{aligned}
& v_{x, B}=d_{B} \cos \theta_{h} \\
& v_{y, B}=d_{B} \sin \theta_{h}
\end{aligned}
$$

Note that $d_{B}=d_{B 1}=d_{B 2}$ and $d_{A}=d_{A 1}=d_{A 2}$ from the constant velocity assumption. Since the position of platform $A$ can be propagated using the on board navigation system of platform $A$, the velocity of platform $A$ does not need to be calculated in this way. At this point, the position of platform $B$ in Cartesian coordinates, $\left(\hat{x}_{B}, \hat{y}_{B}\right)$, will be propagated using the most recent estimates for each of the four solutions. The result is presented in Equation (5.3) and Equation (5.4) where $k$ is the location number, $i$ is the solution number, $\Delta t$ is the change in time between locations, and $v_{x, B}$ and $v_{y, B}$ is the velocity in the $x$ and $y$ directions respectively.

$$
\begin{aligned}
& \hat{x}_{B, k+1}^{(i)}=\hat{x}_{B, k}^{(i)}+v_{x, B} \Delta t \\
& \hat{y}_{B, k+1}^{(i)}=\hat{y}_{B, k}^{(i)}+v_{y, B} \Delta t
\end{aligned}
$$

To determine the weights for each solution, the expected distance between platforms for each solution is calculated using the propagated position of platform $B$. Let $\hat{d}_{i, k}$ be the expected distance for the $i$ th solution at time step $k$ where $\left(\hat{x}_{A, k}, \hat{y}_{A, k}\right)$ and $\left(\hat{x}_{B, k}, \hat{y}_{B, k}\right)$ are the position in Cartesian coordinates for platform $A$ and platform $B$, respectively.

$$
\hat{d}_{k}=\sqrt{\left(\hat{x}_{A, k}-\hat{x}_{B, k}\right)^{2}+\left(\hat{y}_{A, k}-\hat{y}_{B, k}\right)^{2}}
$$

Then, the residual distance, $\tilde{d}$, at time step $k$ can be calculated using the difference in the expected distance and the range measurement.

$$
\tilde{d}_{k}=\hat{d}_{k}-d_{k}
$$


If the system is free of noise, $\tilde{d}=0$ for the true solution and $\tilde{d} \neq 0$ for false solutions. The weights for each solution are assigned as the inverse of the residual distance.

$$
w=\frac{1}{\tilde{d}_{k}}
$$

In practice, the weight with the highest value is chosen as the best solution. If the system is free of noise, (5.7) approaches infinity only for the correct solution provided the constraints are satisfied for the cooperative maneuver. This details of the cooperative maneuver are provided in the following section.

\subsubsection{Constraints for the Cooperative Maneuver}

The residual distance is based on the assumption that the position of none of the four solutions will be equidistant from the platform $A$. Although this seems unlikely, it is possible that the maneuver will result in equidistant solutions, but this can be prevented by checking the trajectory of each maneuver and modifying the maneuver in the situation where the correct solution will be unknown. This is important to check because if equidistant solutions exist after the maneuver, then the correct solution will not be observable. The maneuver will require two constraints in order to guarantee none of the solutions will be equidistant from the platform $A$.

First, the new location of platform $A$ must not lie along the line of the previous trajectory. This constraint guarantees that after the maneuver, only two solutions will remain on one of the sides of the trajectory of platform $A$. This case is illustrated in Figure 5.2. If the fourth location does lie along the line of the previous trajectory, then there will be one solution remaining on each side of platform A. This case is illustrated in Figure 5.1. This is because the pair of solutions corresponding to $-\theta_{h}$ are equivalent to the solutions corresponding to $+\theta_{h}$ reflected across the path of platform $A$. This means that if the new location of platform $A$ is on the side of the pair of solutions corresponding to $-\theta_{h}$, then the residual distance will decrease for $-\theta_{h}$ and increase for $+\theta_{h}$. If the new location of platform $A$ is on the side of the pair corresponding to $+\theta_{h}$, then the residual distance will increase for $-\theta_{h}$ and decrease for $+\theta_{h}$. Therefore, $+\theta_{h}$ or $-\theta_{h}$ can be elimated as long as the fourth location of platform $A$ does not lie along the same line as the previous trajectory.

Second, the new location of platform $A$ must not lie on the perpendicular bisector of the line segment connecting the remaining two solutions. Note that these solutions corresponding to either $+\theta_{h}$ or $-\theta_{h}$. This guarantees that the weights of the remaining solutions will not converge to the same value. The perpendicular bisector of a line segment $P Q$ is a line perpendicular to $P Q$ and 


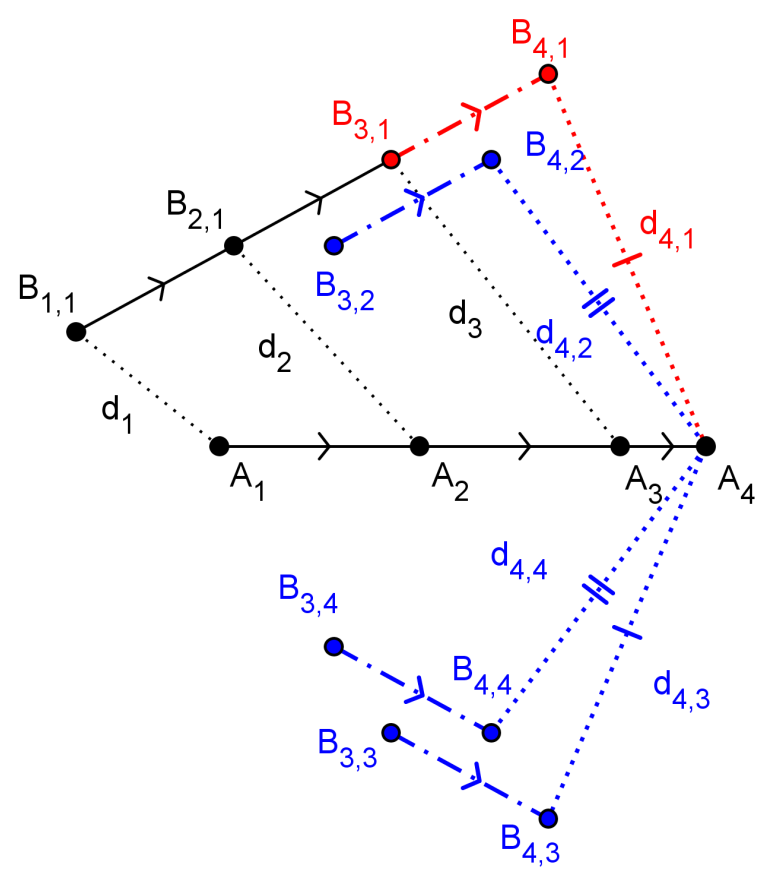

Figure 5.1: Illustration of propagating the position of platform $B$ to a fourth location if platform $B$ remains on the current trajectory and platform $A$ changes trajectories, so that the fourth location is on the same line as the previous trajectory. Red indicates the true solutions, and blue indicates the false solutions. Note that in this case $\operatorname{dist}\left(B_{4,1}, A_{4}\right)=\operatorname{dist}\left(B_{4,3}, A_{4}\right)$ and $\operatorname{dist}\left(B_{4,2}, A_{4}\right)=$ $\operatorname{dist}\left(B_{4,4}, A_{4}\right)$ are equivalent.

passing through the midpoint of $P Q$. In addition, the locus of all points equidistant from the points $P$ and $Q$ lie along the perpendicular bisector of $P Q$. Therefore, if the new location of the platform $A$ is on the perpendicular bisector of the line passing through the remaining two solutions, then platform $A$ will be equidistant from the remaining two solutions. This means the residual distance will be equivalent for the remaining solutions, so no information will be gained, and the correct solution will remain unknown. In order to avoid this situation, the perpendicular bisector can simply be calculated for the remaining solutions, and the trajectory can be verified before performing the maneuver. Let the remaining two solutions be denoted in Cartesian coordinates by the points $P$ $=\left(\hat{x}_{B, k+1}^{(1)}, \hat{y}_{B, k+1}^{(1)}\right)$ and $Q=\left(\hat{x}_{B, k+1}^{(2)}, \hat{y}_{B, k+1}^{(2)}\right)$ and let the line segment connecting the two solutions be denoted as $P Q$. The perpendicular bisector passing through $P Q$ is presented in Figure 5.3. The perpendicular bisector of the line segment $P Q$ is straightforward to calculate given $P$ and $Q$. The perpendicular bisector passes through the midpoint of $P Q$, and the slope of the perpendicular bisector is simply the negative reciprocal of the slope of $P Q$. The midpoint of $P Q$ is denoted by 


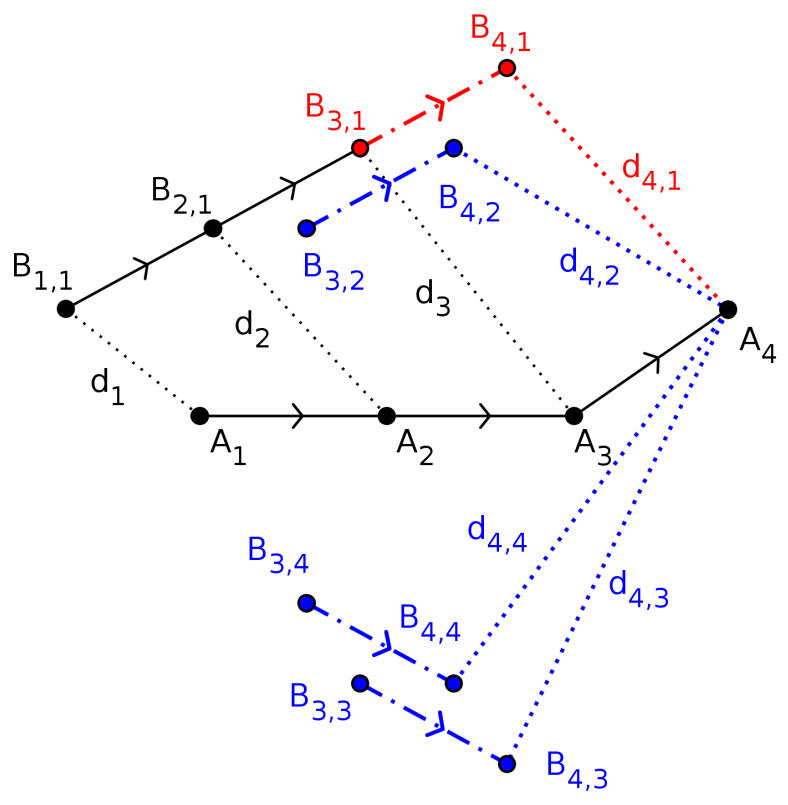

Figure 5.2: Illustration of propagating the position of platform $B$ to a fourth location if the platform $B$ remains on the current trajectory and platform $A$ changes trajectories, so that the fourth location does not like on the same line as the previous trajectory. Red indicates the true solutions, and blue indicates the false solutions. Note that in this case $\operatorname{dist}\left(B_{4,1}, A_{4}\right) \neq \operatorname{dist}\left(B_{4,2}, A_{4}\right) \neq \operatorname{dist}\left(B_{4,3}, A_{4}\right) \neq$ $\operatorname{dist}\left(B_{4,4}, A_{4}\right)$. In addition, $\operatorname{dist}\left(B_{4,3}, A_{4}\right)$ and $\operatorname{dist}\left(B_{4,4}, A_{4}\right)$ are both greater than $\operatorname{dist}\left(B_{4,1}, A_{4}\right)$ and $\operatorname{dist}\left(B_{4,2}, A_{4}\right)$

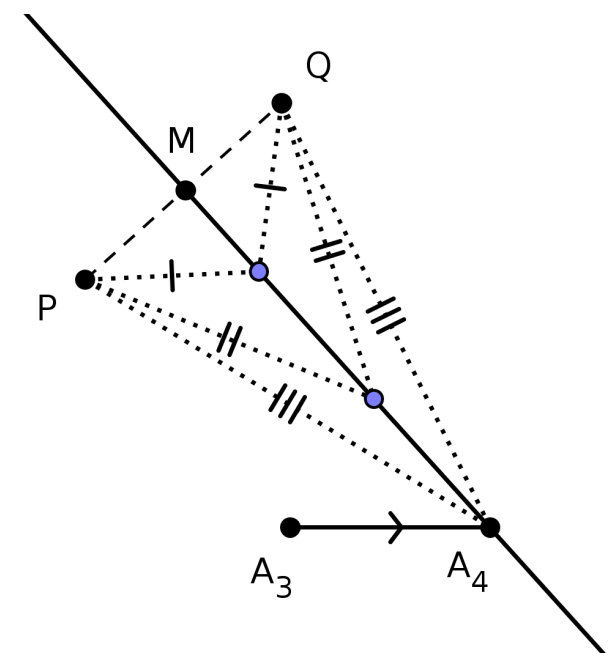

Figure 5.3: Perpendicular bisector of the line $P Q$. The locus of all points equidistant from $P$ and $Q$ are contained on the perpendicular bisector of $P Q$. In the figures, the fourth location of $A$ is along the same trajectory of the first three locations.

$M=\left(m_{x}, m_{y}\right)$ and is given in Equation (5.8).

$$
\left(m_{x}, m_{y}\right)=\left(\frac{\hat{x}_{B, k+1}^{(1)}+\hat{x}_{B, k+1}^{(2)}}{2}, \frac{\hat{y}_{B, k+1}^{(1)}+\hat{y}_{B, k+1}^{(2)}}{2}\right)
$$


The slope of $P Q$ is denoted by $s$ and is given in Equation (5.9).

$$
s=\frac{\hat{y}_{B, k+1}^{(2)}-\hat{y}_{B, k+1}^{(1)}}{\hat{x}_{B, k+1}^{(2)}-\hat{x}_{B, k+1}^{(1)}}
$$

Now, the line that defines the perpendicular bisector of $P Q$ can be written in terms of the $m_{x}, m_{y}$, and $s$. The result is given in Equation (5.10).

$$
y=-\frac{1}{s}\left(x-m_{x}\right)+m_{y}
$$

Using the perpendicular bisector of $P Q$, it can be verified that the new location of platform $A$ will not be equidistant to $P$ and $Q$. This can be done by rearranging Equation (5.10) to obtain Equation (5.11) and substituting the position of platform $A$ for $x$ and $y$ where $\epsilon$ is a very small number.

$$
\left|y-\left(-\frac{1}{s}\left(x-m_{x}\right)+m_{y}\right)\right|>\epsilon
$$

If Equation (5.11) is satisfied, then the location of platform $A$ will be equidistant from each of the remaining solutions. In this case, the platform can modify the maneuver until Equation (5.11) is no long satisfied. Note that $\epsilon$ can be increased or reduced based on the accuracy of the estimation algorithm. Due to noisy measurements, a large $\epsilon$ may be required in order to move the location further from the perpendicular bisector. As the location move further from the perpendicular bisector, there will be a more clear distinction between the weights of the remaining two solutions. This is because along the perpendicular bisector, the remaining two solutions are equidistant from platform $A$, so the weights are equivalent since the residual distances are equivalent. As platform $A$ moves away from the perpendicular bisector, the remaining two solutions are no longer equidistant, so the weights will no longer be equivalent, and the difference between the weights for the remaining solutions will increase as the platform moves further from the perpendicular bisector. Note that the residual distance will never be greater than the distance between $P$ and $Q$, so regardless of the maneuver, the residual distance will be in the interval $0<\tilde{d}_{k}<\operatorname{dist}(P, Q)$ for the remaining two solutions. 


\section{Chapter 6}

\section{Noisy Measurements}

In this chapter, the case is considered where the platforms are traveling along intersecting lines, so there will be four potential solutions for the relative pose. If the system is free of noise, each of the solutions for the relative pose can simply be obtained by plugging in the range measurements and displacement in Equation (4.18) and Equation (4.25). Since the range measurements and displacement are both noisy, the result may be inaccurate or unsolvable depending on the magnitude of the noise in the measurements. Since the distance between platforms is provided directly by the measurements, only the relative heading and relative bearing of platform $B$ need to be estimated in order to obtain the relative pose between platforms. In this section, a simple method will be presented for estimating the relative pose for each of the four solutions, then a sensitivity analysis will be performed on results of the estimation strategy. Finally, the accuracy and reliability of the cooperative maneuver will be analyzed for various magnitudes of measurement noise.

\subsection{Estimating the Relative Pose}

Since the platforms are traveling at a constant velocity, the angle between the trajectories, $\theta_{h}$, is constant over any number of time steps. Due to this, a system of equations for $\theta_{h}$ can be formed 
using $n$ observations. Letting

$$
X_{h}=\left[\begin{array}{c}
r_{a}\left(d_{1}, d_{2}, d_{3}\right) \\
r_{a}\left(d_{2}, d_{3}, d_{4}\right) \\
\cdot \\
\cdot \\
r_{a}\left(d_{n-2}, d_{n-1}, d_{n}\right)
\end{array}\right]
$$

and

$$
Y_{h}=\left[\begin{array}{c}
r_{b}\left(d_{1}, d_{2}, d_{3}\right) \\
r_{b}\left(d_{2}, d_{3}, d_{4}\right) \\
\cdot \\
\cdot \\
r_{b}\left(d_{n-2}, d_{n-1}, d_{n}\right)
\end{array}\right]
$$

, the system can be represented as in Equation (6.3).

$$
X_{h} \sin \theta_{h}=Y_{h}
$$

Using linear least squares, a batch estimate of $\sin \theta_{h}$ can be obtained using $n$ observations as presented in Equation (6.4).

$$
\sin \theta_{h}=\left(X_{h}^{T} X_{h}\right)^{-1} X_{h}^{T} Y_{h}
$$

Note that each observation includes three measurements, so the relative position cannot be estimated without at least three measurements. For example, observation at time step $k$ includes measurements from $k, k-1$, and $k-2$. In addition, the least square estimate of $\theta_{h}$ is sensitive to the geometry and noise. The sensitivity will be discussed later in this chapter. Applying the quadratic formula to Equation (4.25), each of the four solutions can be estimated independently using nonlinear least squares. The relative bearing using the quadratic formula is presented in Equation (6.5) where $\alpha$, $\beta$, and $\gamma$ are all functions of $\pm \theta_{h}$.

$$
2 \alpha t=-\beta \pm \sqrt{\beta^{2}-4 \alpha \gamma}
$$


Similar to $\theta_{h}$, a system of equations for $\theta_{b}$ can be formed using $n$ observations. Letting

$$
X_{b}=\left[\begin{array}{c}
(2 \alpha)_{1} \\
(2 \alpha)_{2} \\
\cdot \\
\cdot \\
(2 \alpha)_{n}
\end{array}\right]
$$

and

$$
Y_{b}=\left[\begin{array}{c}
\left(-\beta \pm \sqrt{\beta^{2}-4 \alpha \gamma}\right)_{1} \\
\left(-\beta \pm \sqrt{\beta^{2}-4 \alpha \gamma}\right)_{2} \\
\cdot \\
\cdot \\
\left(-\beta \pm \sqrt{\beta^{2}-4 \alpha \gamma}\right)_{n}
\end{array}\right]
$$

, the systems can be represented as in Equations (6.8) where $t=\tan \theta_{2} / 2$.

$$
X_{b} t=Y_{b}
$$

Using linear least squares, a batch estimate of $t=\tan \theta_{2} / 2$ can be obtained using $n$ observations as presented in Equations (6.9).

$$
t=\left(X_{b}^{T} X_{b}\right)^{-1} X_{b}^{T} Y_{b}
$$

Note that $\alpha, \beta$, and $\gamma$ are all functions of $\theta_{h}$, the range measurements, and the displacement between locations of each platform, so $\theta_{b}=f\left( \pm \theta_{h}\right)$, and $\theta_{h}$ must be estimated prior to $\theta_{b}$. Since the relative bearing changes at each time step, a batch estimate of the relative bearing may only be performed at certain geometries. Since the formulation is sensitive to noise, the solutions are smoothed using exponential smoothing as presented in Equation (6.10) where $\delta$ is the smoothing factor.

$$
\theta_{b, k}=\delta \theta_{b, k-1}+(1-\delta) \theta_{b, k-1}
$$

In the case $\beta^{2}-4 \alpha \gamma<0$, additional measurements can be obtained to solve for the relative bearing. In addition, it should be noted that $\theta_{b}$ is not constant, but the change in $\theta_{b}$ is negligible in most 
cases, so the number of observation should be limited when performing a batch estimate of $\theta_{b}$. Since $\theta_{h}$ is constant, any number of observations can be used for the batch estimate of $\theta_{h}$.

\subsection{Sensitivity Analysis}

Here only the case is considered where the platforms are traveling along intersecting lines, as the other three cases are not difficult to detect and are not as likely in most scenarios. The simulations were performed by adding zero mean Gaussian noise to the distance measurements between platforms and the displacement in position between locations. In performing various simulations, the goal is to verify the algorithm and analyze the sensitivity to different parameters. Note that in the sensitivity analysis $\theta_{h}$ will be referred to as $\theta_{3}$ and $\theta_{b}$ will be referred to as $\theta_{2}$. The first simulation experiment consists of a single path for platform $A$ and platform $B$. In this simulation experiment, the platforms start in different locations 150 meters apart. The distance intervals $d_{A}$ and $d_{B}$ between observations is varied to analyze the sensitivity to this parameter while the level of noise in the ranging measurement and displacement are both held constant. The level of ranging error for displacement has a standard deviation between $1 \%$ and $5 \%$ the total displacement, which is based on the typical performance of current visual odometry algorithms $[34,35,36,37]$. The average root mean square error (RMSE) for the relative heading and relative bearing is presented in Fig. 6.1 for 100 Monte Carlo trials for each distance interval. As the distance interval increases, the error in both the relative heading and relative bearing decrease. This is likely due to the effect of the distance interval on the ratio of the displacement in position to the distance between platforms, $r_{2} / r_{1}$. If $r_{2} / r_{1}$ is small, the number of observation included in the least squares estimate has little effect on the accuracy. This means that only a few measurements are necessary to obtain an accurate estimate if $r_{2} / r_{1}$ is small. If the platforms are far away from each other, the time between observations must be increased to obtain a larger distance interval and lower $r_{2} / r_{1}$, so each platform must travel a straight line path for a longer amount of time if the platforms are far away from each other. In the case the platforms are flying in directions which increase the ratio over time, the batch size can be increased to reduce drift and variance by using additional observations when $r_{2} / r_{1}$ is small. Note that $r_{2} / r_{1}$ always increases over time if the platforms are traveling away from each other. This is because the distance interval remains constant while the distance between platforms increase. Since the platforms are required to travel at constant velocity for this method, $r_{2} / r_{1}$ is guaranteed to increase if the platforms are traveling away from each other. This issue can be mitigated by performing a

batch estimate which includes observations when $r_{2} / r_{1}$ is small. Since $r_{2} / r_{1}$ is dependent on the 
distance interval, similar performance can be obtained at large distances by increasing the distance interval to reduce $r_{2} / r_{1}$. The cumulative distribution function (CDF) for the trials are presented in Fig. 6.2.
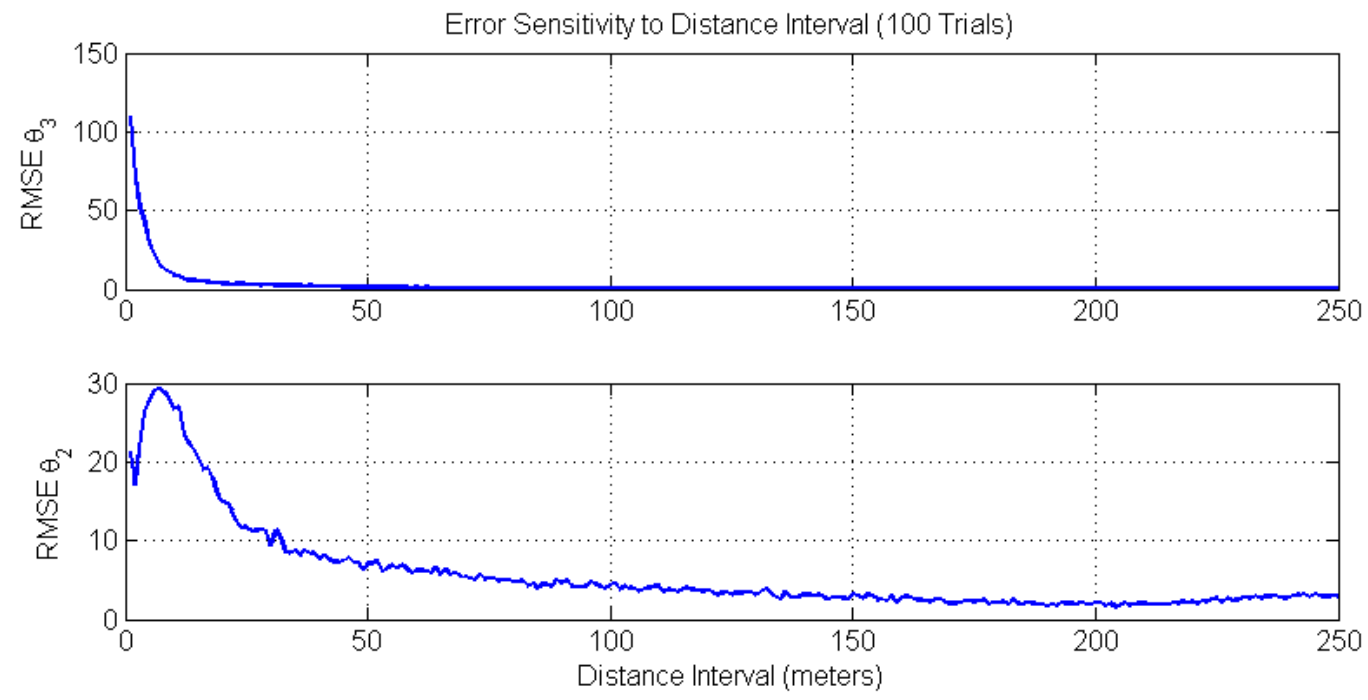

Figure 6.1: Average RMSE for the relative heading and relative bearing of platform $B$. The horizontal axis is the distance interval, and the vertical axis is the average RMSE error in degrees. 100 trials were completed for each distance interval.
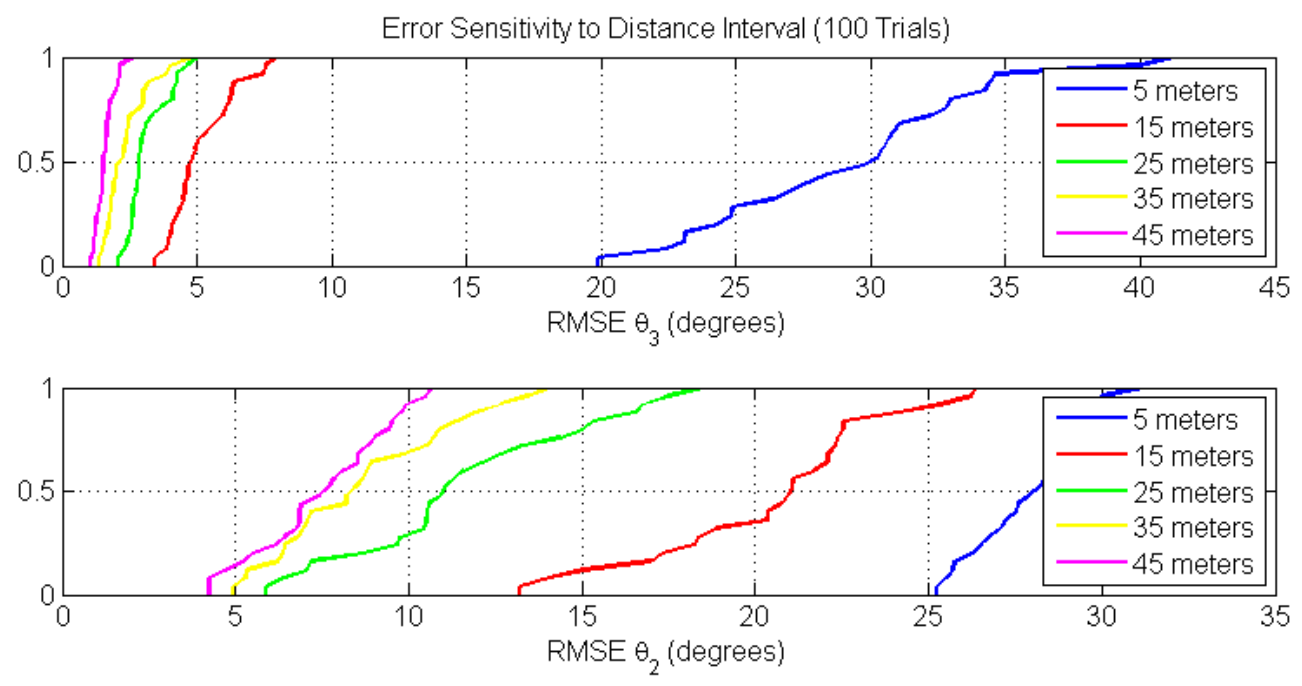

Figure 6.2: CDF of the relative heading and relative bearing of platform $B$ with respect to platform $A$ for various distance intervals. 100 trials were completed for each distance interval.

In the next simulation experiment, the distance interval is held constant at 25 meters. The level of noise in the ranging measurement is held constant with a standard deviation of 0.05 meters, and the level of noise in the displacement in position is held constant at $1.7 \%$ of the displacement. The 
flight path is varied by changing the heading of platform $B$ with respect to platform $A$ to analyze the sensitivity to flight path geometry. The RMSE of the relative heading and relative bearing are presented in Fig. 6.3. The error for the relative heading is greatest when the platform trajectories are nearly parallel. Although a parallel configuration is detectable from the distance measurements, the error in the relative position is still greatest in this configuration. The CDF for the trials are presented in Fig. 6.4. The performance is similar for the platforms when the trajectories are not near parallel with less than 1 degree error for the relative heading and less than 5 degrees error for the relative bearing in $100 \%$ of the trials at 45,90 , and 135 degrees for the relative heading, but the performance decreases quickly as the relative heading approaches 0 and 180 degrees.
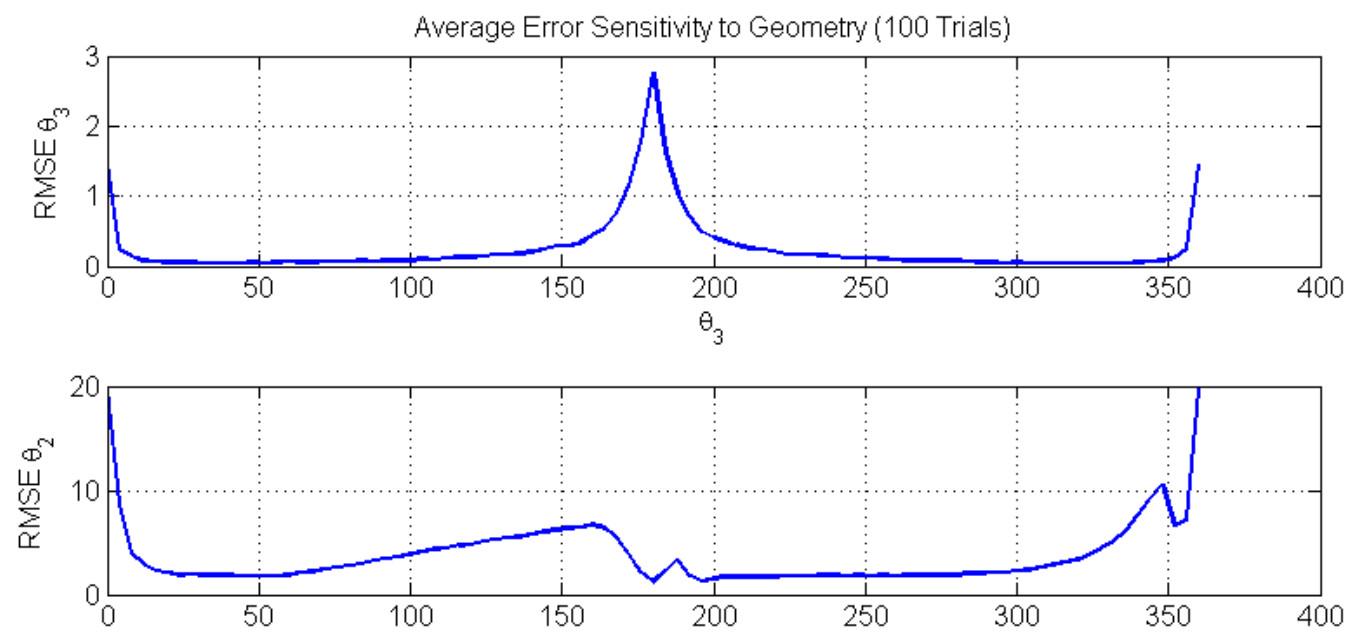

Figure 6.3: Average RMSE for the relative heading and relative bearing of platform $B$. The horizontal axis is the relative heading of platform $B$, and the vertical axis is the average RMSE error in degrees. 100 trials were completed for each relative heading of platform $B$.

In the next simulation experiment, the distance interval is held constant at 25 meters, and the geometry is held constant with the relative heading of platform $B$ at 0 degrees. The level of noise for the ranging measurement is varied based on high and low quality ranging sensors. The level of noise for the displacement in position is held constant with a standard deviation of $1.7 \%$ of the displacement. The RMSE of the relative heading and relative bearing are presented in Fig. 6.5. The CDF for the trials are presented in Fig. 6.6.

In the next simulation experiment, the distance interval is held constant at 25 meters, and the geometry is held constant with the relative heading of platform $B$ at 0 degrees. The standard deviation for the displacement in position is varied between $1 \%$ and $5 \%$ of the total displacement. The level of noise for the ranging measurements is held constant with a standard deviation of 0.05 

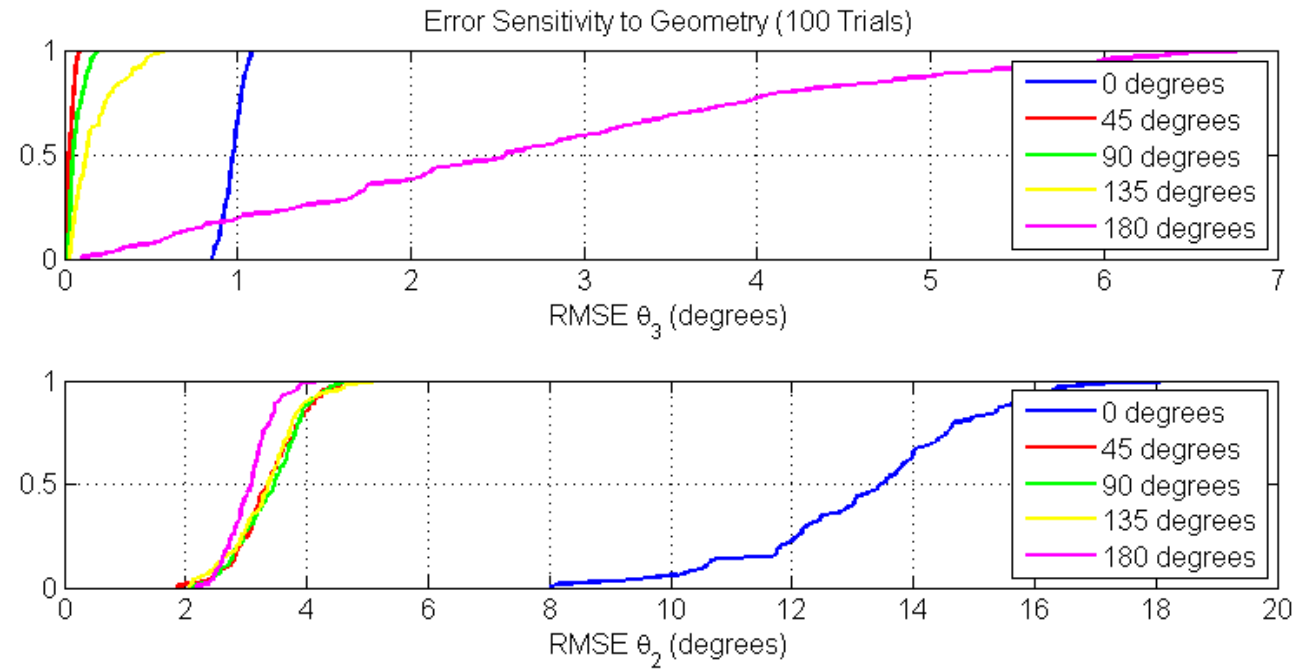

Figure 6.4: CDF of the relative heading and relative bearing of platform $B$ with respect to platform $A$ for various relative headings of platform $B .100$ trials were completed for each relative heading of platform $B$.
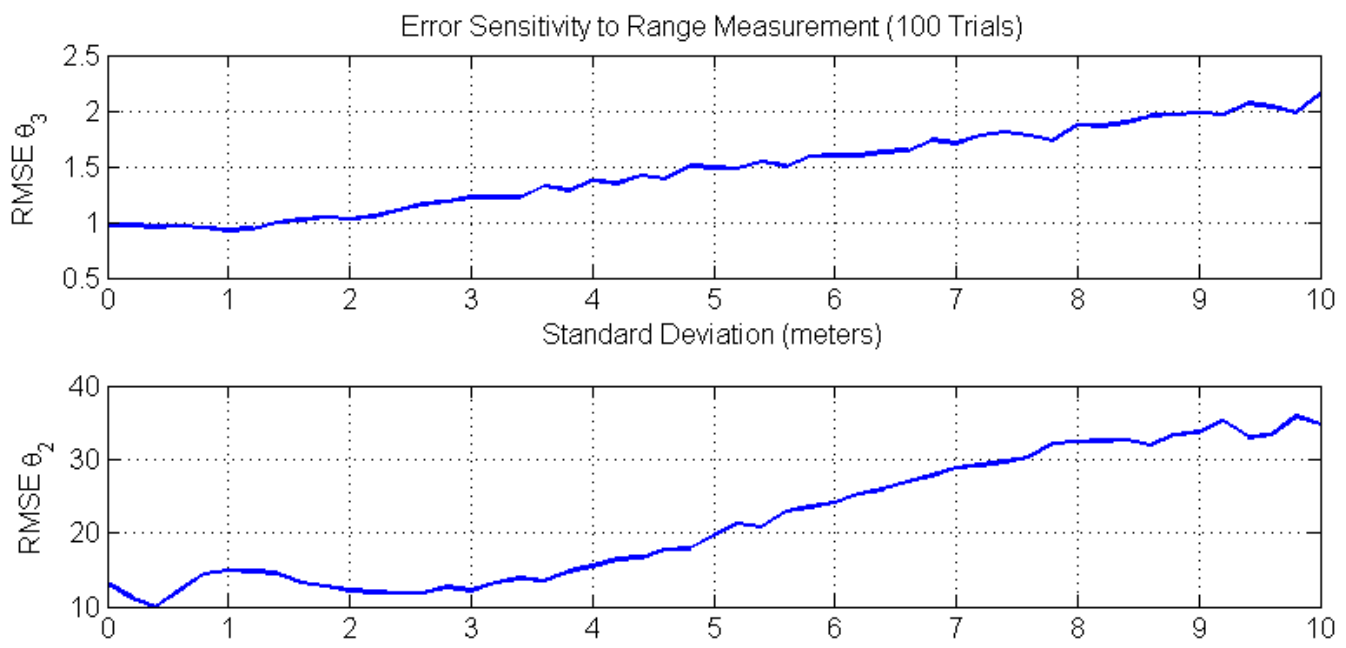

Figure 6.5: Average RMSE for the relative heading and relative bearing of platform $B$. The horizontal axis is the standard deviation of ranging measurements between platforms, and the vertical axis is the average RMSE error in degrees. 100 trials were completed for each level of ranging error.

meters. The RMSE of the relative heading and relative bearing are presented in Fig. 6.7. The CDF for the trials are presented in Fig. 6.8. 

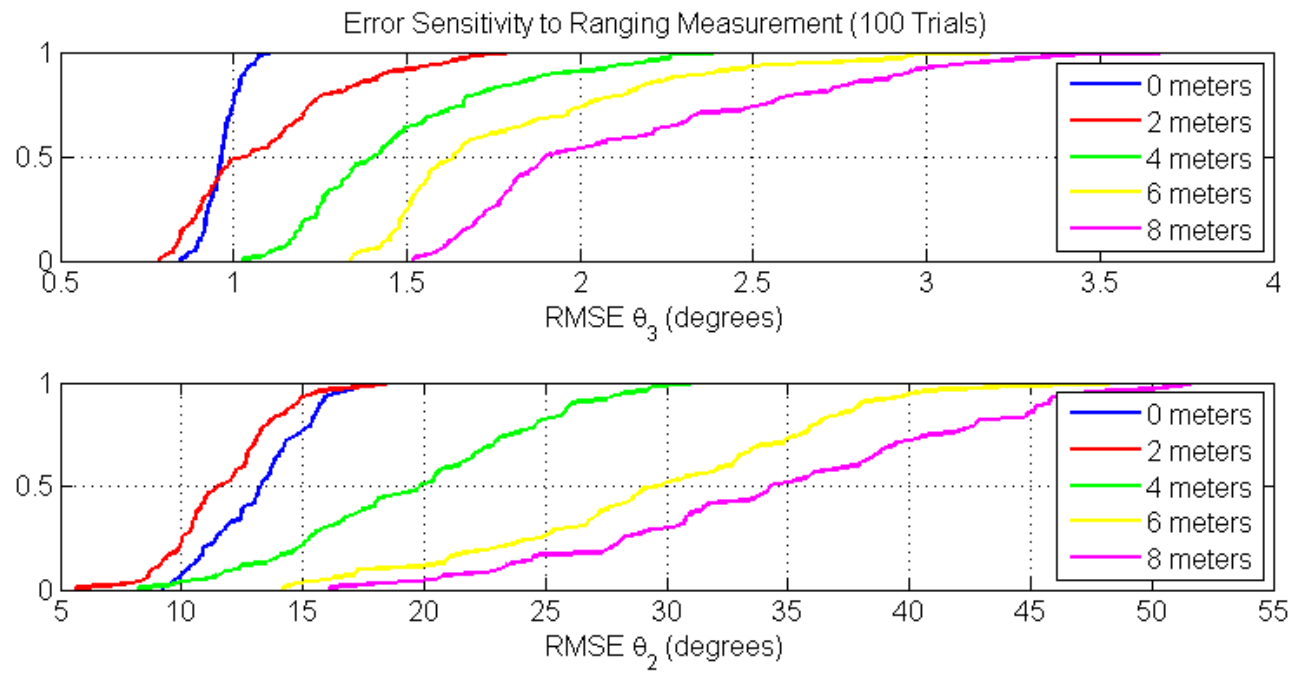

Figure 6.6: $\mathrm{CDF}$ of the relative heading and relative bearing of platform $B$ with respect to platform $A$ for various levels of error for the ranging measurements between platforms. 100 trials were completed for each level of ranging error.
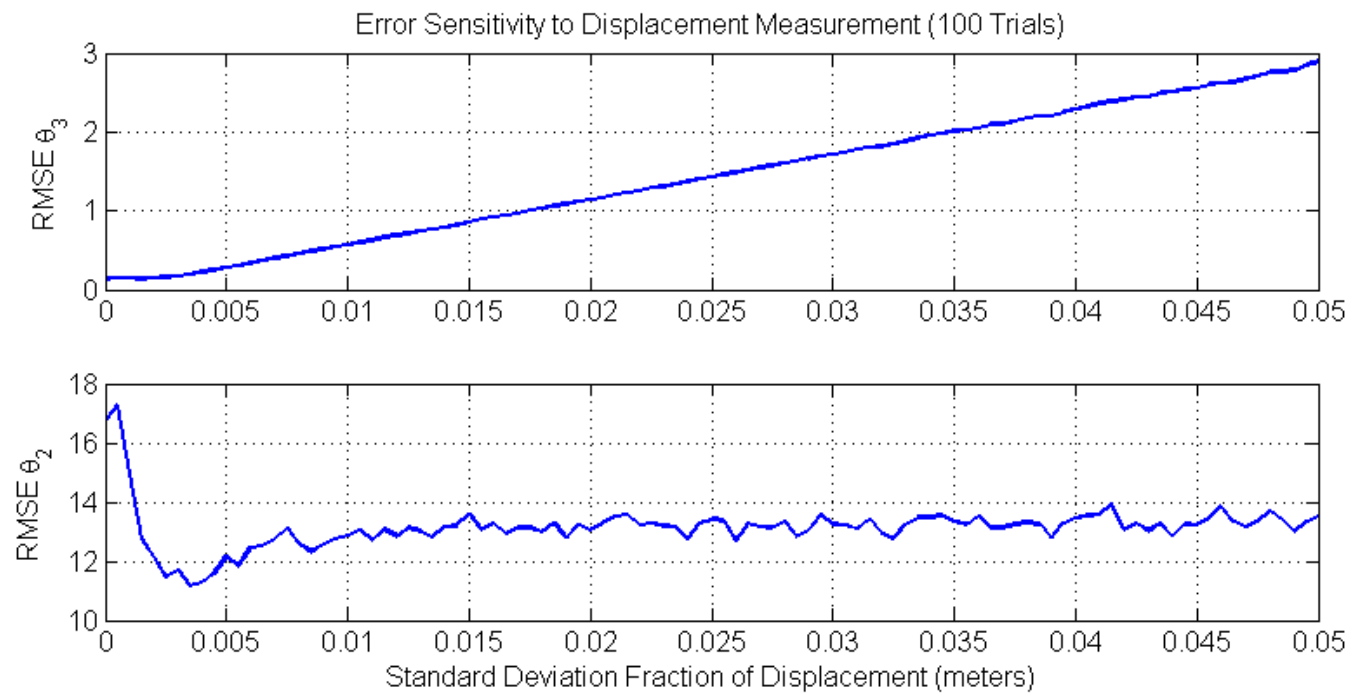

Figure 6.7: Average RMSE for the relative heading and relative bearing of platform $B$. The horizontal axis is the standard deviation as a fraction of the total displacement between locations, and the vertical axis is the average RMSE error in degrees. 100 trials were completed for each level of displacement error. Note the error is shown as the percentage of the displacement.

\subsection{Reduction Accuracy}

In the last simulation experiment, the reduction of solutions is analyzed for various geometries and noise levels. The objective of this simulation experiment is to verify the validity of the algorithm for reducing the number of solutions. The distance interval is held constant at 25 meters for this simulation experiment, but the geometry and initial position of each platform are varied for each 

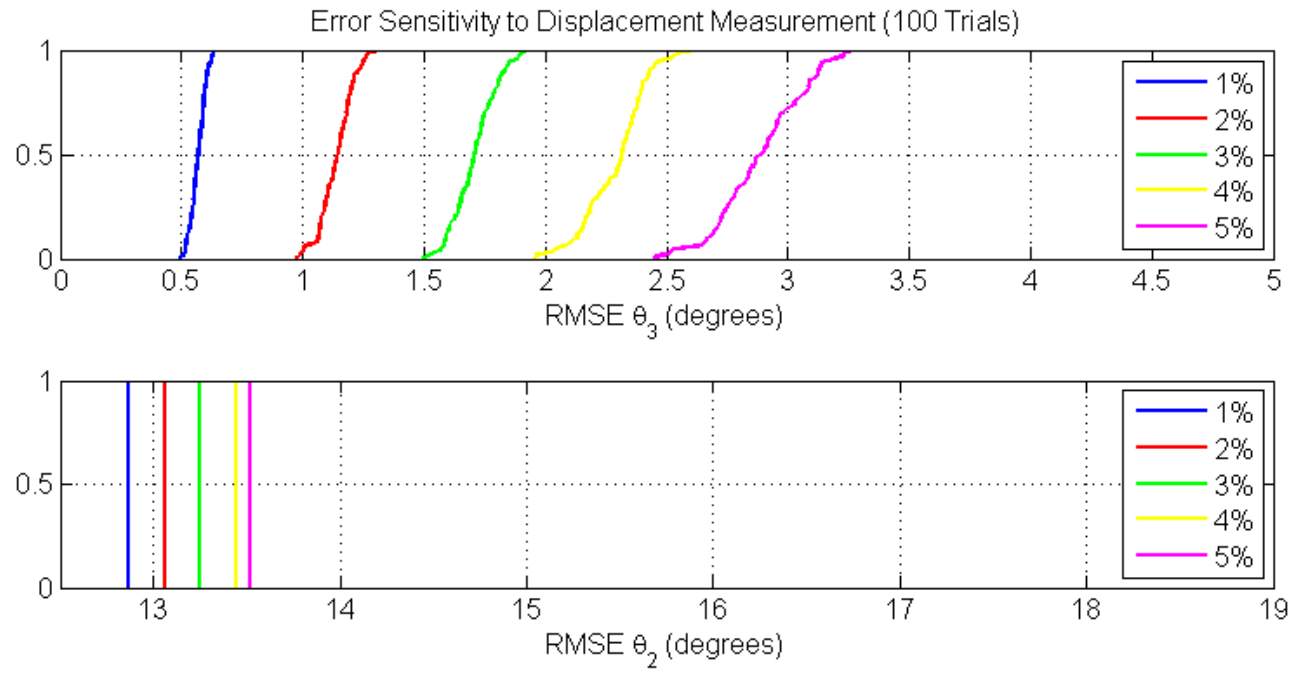

Figure 6.8: CDF of the relative heading and relative bearing of platform $B$ with respect to platform $A$ for various levels of error for the displacement in position between locations. 100 trials were completed for each level of displacement error. Note the standard deviation of the displacement are shown as a percent of the total displacement.

simulation. The starting position of each platform are randomly chosen according to a uniform distribution within a 1000 by 1000 meters square.

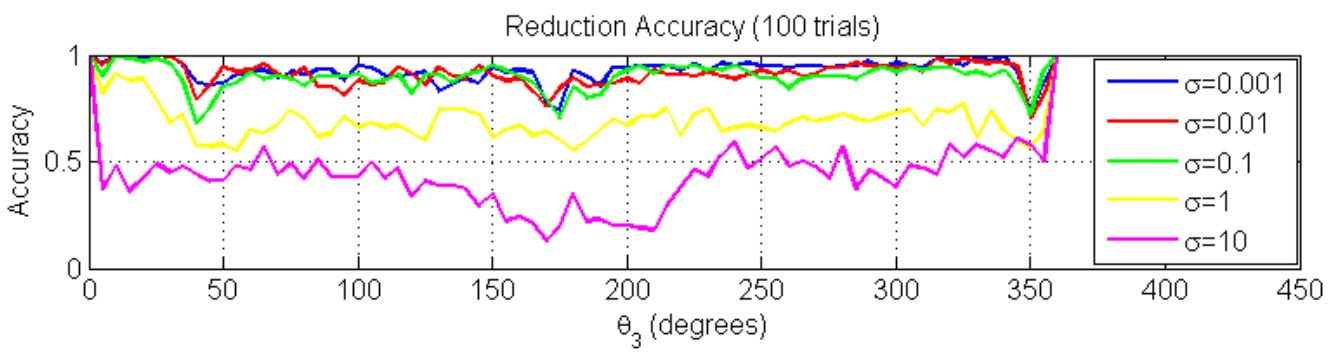

Figure 6.9: Percentage of trials that successfully predicted the correct solution for various levels of error for the ranging error. The horizontal axis is the heading of platform $B$ and the vertical axis is the percentage of trials that successfully predicted the correct solution.

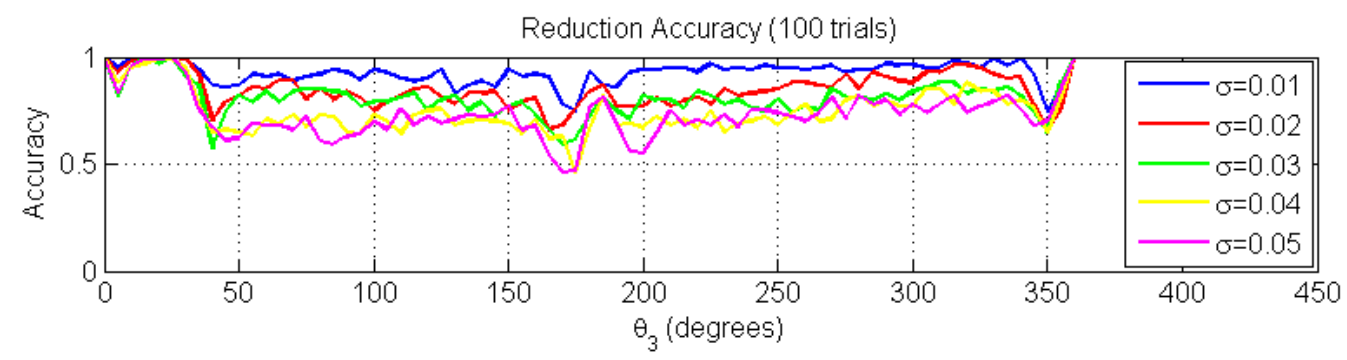

Figure 6.10: Percentage of trials that successfully predicted the correct solution for various levels of error for the displacement error. The horizontal axis is the heading of platform $B$ and the vertical axis is the percentage of trials that successfully predicted the correct solution. 
As seen in Figure 6.9 and Figure 6.10, the relative heading of platform $B$ does not affect the accuracy of predicting the correct solution except when the platforms are traveling parallel. In this case, the correct solution is predicted in $100 \%$ of trials. When the platforms are not traveling parallel, the level of error in the ranging measurements and displacement in position greatly affect the outcome of the algorithm. If the error is too large, the algorithm may predict the incorrect solution. This is due to the fact that the residual distance is calculated by propagating the position obtained from relative bearing. If the error in the relative bearing is too large, the residual distance may not be accurate, which results in a false detection of the correct solution. 


\section{Chapter 7}

\section{Concluding Remarks}

This thesis presents an algorithm for estimating the relative pose between two moving UAVs using range-only measurements and analyzes the sensitivity to flight geometry, observation frequency, and measurement noise. Using this method, the maximum number of solutions for the relative pose given three aircraft-to-aircraft range measurements is shown to be at most four, and a cooperative maneuver was presented in order to obtain a single unique solution. The specific method for localizing each aircraft does not affect the algorithm, so visual odometry, inertial navigation, or landmark based navigation can all be used for this method.

\subsection{Discussion of Results}

To analyze the effectiveness of the developed algorithm, several Monte Carlo simulations were completed through varying the observation frequency, trajectory geometry, and measurement noise. The simulations show that the algorithm is most sensitive to the ratio of the aircraft-to-aircraft range measurements over the displacement in position between observations. In order to mitigate this problem, the sensitivity can be reduced by increasing the distance interval. Furthermore, the accuracy could potentially be increased by estimating the relative pose using a recursive filter to continuously update the relative bearing since this value is not constant. The largest disadvantage of using the presented estimation method is that the relative bearing is not constant between observations; therefore, only a small number of observations can be used in the batch estimate of the relative bearing. In contrast, the relative heading is constant between observation, so a high number of observation can be used to obtain reliable estimate of the relative heading. 


\subsection{Contribution}

This work contrasts with the majority methods for the relative localization, which aim to solve the problem for arbitrary motion rather than coordinated motion. The main contribution of this work is the enabling of relative localization through coordinated movement. In addition, the problem is solved using range-only measurements and the displacement in position of each UAV. The majority of cooperative localization problems require knowledge of the relative pose between UAVs, and in absence of GPS or landmark reference, the relative pose is unknown. The proposed method solves this problem by enforcing constraints on the motion of the UAVs while collecting measurements, which allows the relative pose to be obtained with only four sets of measurements. Using three set of measurements, it is shown that at most four solutions exist if the platforms fly at constant velocities. In addition, it is shown that if a single maneuver is performed to collect a fourth set of measurements, a unique solution can be obtained for the relative pose. The primary contribution of this work is the leveraging of coordinated motion in order to obtain a unique solution with only four sets of measurements.

\subsection{Future Work}

The work presented in this thesis opens a number of research directions for cooperative localization using coordinated motion. First, the method presented only shows how to guarantee a unique solution, but the optimal solution in order to maximize the likelihood is not discussed. In addition, only the initialization process is presented in this work. After the initialization of the relative pose, a recursive filter could provide a way to continuously estimate the relative pose over a long period of time. Lastly, several practical problems exist for implementation such as communication and measurement noise. Although measurement noise was considered during simulation, the noise models developed for simulation were simplistic and do not guarantee the same performance in experimental testing as in simulation. Therefore, future work will include system development for experimental testing. 


\section{Bibliography}

[1] J. Hardy, J. Strader, J. N. Gross, Y. Gu, M. Keck, J. Douglas, C. N. Taylor, Unmanned Aerial Vehicle Relative Navigation in GPS Denied Environments in Proceedings of IEEE/ION Position Localization and Navigation Symposium. IEEE/ION, 2016.

[2] J. Strader, Y. Gu, J. Gross, M. DePetrillo, and J. Hardy, Cooperative relative localization for moving uavs with single link range measurements, in Proceedings of IEEE/ION Position Location and Navigation Symposium. IEEE/ION, 2016.

[3] Bahr, Alexander, John J. Leonard, and Maurice F. Fallon. Cooperative localization for autonomous underwater vehicles. The International Journal of Robotics Research 28.6 (2009): 714-728.

[4] M. Betke and L. Gurvits. Mobile robot localization using landmarks. In Proceedings of the IEEE International Conference on Robotics and Automation, volume 2, pages 135142, Apr. 1994. See also IEEE Transactions on Robotics and Automation, 13:2, pp. 251-263, 1997.

[5] L. Kleeman. Optimal estimation of position and heading for mobile robots using ultrasonic beacons and dead-reckoning. In Proceedings of the IEEE International Conference on Robotics and Automation, pages 25822587, Nice, France, 1992.

[6] J. Wang, R. Ghosh, and S. K. Das, A survey on sensor localization, J. Control Theory Appl., vol. 8, no. 1, pp. 211, 2010.

[7] A. C. Sanderson, A distributed algorithm for cooperative navigation among multiple mobile robots, Advanced Robotics, vol. 12, no. 4, pp. 335-349, 1998

[8] R. Kurazume and S. Hirose, Study on cooperative positioning system: optimum moving strategies for CPS-III, in Proceedings of the IEEE International Conference on Robotics and Automation, Leuven, Belgium, May 16-20, 1998, pp. 2896-2903. 
[9] R. Kurazume and S. Hirose, An experimental study of a cooperative positioning system, Autonomous Robots, vol. 8, no. 1, pp. 43-52, Jan. 2000

[10] R. Kurazume, S. Hirose, S. Nagata, and N. Sashida, Study on cooperative positioning system (basic principle and measurement experiment), in Proceedings of the IEEE International Conference on Robotics and Automation, Minneapolis, MN, Apr. 22-28, 1996, pp. 1421-1426

[11] R. Kurazume, S. Nagata, and S. Hirose, Cooperative positioning with multiple robots, in Proceedings of the IEEE International Conference on Robotics and Automation, San Diego, CA, May 8-13, 1994, pp. 1250-1257

[12] A. I. Mourikis and S. I. Roumeliotis, Performance analysis of multirobot cooperative localization, IEEE Transactions on Robotics, vol. 22, no. 4, pp. 666681, Aug. 2006

[13] R. Grabowski and P. Khosla, Localization techniques for a team of small robots, in Proceedings of the IEEE/RSJ International Conference on Intelligent Robots and Systems, Maui, HI, Oct. 29 - Nov. 3, 2001, pp. 1067-1072.

[14] I. M. Rekleitis, G. Dudek, and E. E. Milios, Multi-robot collaboration for robust exploration, in Proceedings of the IEEE International Conference on Robotics and Automation, vol. 4, San Francisco, CA, Apr. 2000, pp. 31643169

[15] N. Trawny and T. Barfoot, Optimized motion strategies for cooperative localization of mobile robots, in Proc. of the IEEE International Conference on Robotics and Automation, New Orleans, LA, Apr. 26 - May 1, 2004, pp. 1027-1032

[16] A. Howard, M. J. Mataric, and G. S. Sukhatme, Localization for mobile robot teams using maximum likelihood estimation, in Proceedings of the IEEE/RSJ International Conference on Intelligent Robots and Systems, vol. 1, Lausanne, Switzerland, Sept. 30 Oct. 4, 2002, pp. 434439.

[17] S. 1. Roumeliotis and G. A. Bekey. Collective locali2ation: a distributed kalman filter approach. In Proceedings of the IEEElnterwtionol Conference on Robotics ad Automtion. volume 3, pages 2958-2965, San Fransisco, California, 2000.

[18] S. I. Roumeliotis and G. A. Bekey, Distributed multirobot localization, IEEE Transactions on Robotics and Automation, vol. 18, no. 5, pp. 781-795, Oct. 2002.

[19] R. Madhavan, K. Fregene, and L. E. Parker, Distributed heterogeneous outdoor multi-robot localization, in Proceedings of the IEEE International Conference on Robotics and Automation, Washington, DC, May 11-15, 2002, pp. 374-381. 
[20] S. I. Roumeliotis and G. A. Bekey, Distributed multirobot localization," IEEE Transactions on Robotics and Automation, vol. 18, no. 5, pp. 781-795, Oct. 2002.

[21] E. Nerurkar, S. I. Roumeliotis, and A. Martinelli, Distributed maximum a posteriori estimation for multi-robot cooperative localization, in Proc. of the IEEE International Conference on Robotics and Automation, Kobe, Japan, May 12-17, 2009, pp. 1402-1409.

[22] A. Howard, M. J. Mataric, and G. S. Sukhatme, Putting the I in team: an ego-centric approach to cooperative localization, in Proceedings of the IEEE International Conference on Robotics and Automation, Taipei, Taiwan, Sep. 14-19, 2003, pp. 868-874.

[23] N. Karam, F. Chausse, R. Aufr'ere, and R. Chapuis, Localization of a group of communicating vehicles by state exchange, in Proceedings of the IEEE/RSJ International Conference on Intelligent Robots and Systems, Beijing, China, Oct. 9-15, 2006, pp. 519-524.

[24] S. Panzieri, F. Pascucci, and R. Setola, Multirobot localization using interlaced extended Kalman filter. 2006 IEEE/RSJ International Conference on Intelligent Robots and Systems. IEEE, 2006.

[25] L. Glielmo, R. Setola, and F. Vasca, An interlaced extended Kalman filter, IEEE Trans. on Automatic Control, vol. 44, no. 8, pp. 15461549, 1999.

[26] A. Martinelli, Improving the precision on multi robot localization by using a series of filters hierarchically distributed, in Proceedings of the IEEE/RSJ International Conference on Intelligent Robots and Systems, San Diego, CA, Oct. 29 - Nov. 2, 2007, pp. 1053-1058.

[27] X. Zhou and S. Roumeliotis, Robot-to-robot relative pose estimation from range measurements, IEEE Trans. Robot., vol. 24, no. 6, pp. 13791393, Dec. 2008.

[28] N. Trawny and S. I. Roumeliotis, On the global optimum of planar, range-based robot-to-robot relative pose estimation, in ICRA, Anchor-age, AK, 2010, pp. 32003206

[29] I. Shames, B. FIdan, B. D. O. Anderson, and H. Hmam, Cooperative self-localization of mobile agents, To appear in IEEE Transactions on Aerospace and Electronic Systems, 2011.

[30] C. Alejandro and R. Nagpal, Distributed Range-Based Relative Localization of Robot Swarms, Algorithmic Foundations of Robotics XI, pp. 91-107, Springer International Publishing, 2015. 
[31] G. Papadopoulos, M. F. Fallon, J. J. Leonard, and N. M. Patrikalakis, Cooperative localization of marine vehicles using nonlinear state estimation. Intelligent Robots and Systems (IROS), 2010 IEEE/RSJ International Conference on. IEEE, 2010.

[32] Quenzer, Jake D., and Kristi A. Morgansen. Observability based control in range-only underwater vehicle localization. 2014 American Control Conference. IEEE, 2014.

[33] J. Uicker, G. R. Pennock, and J. E. Shigley, Theory of Machines and Mechanisms. New York: Oxford Univ. Press, 2003.

[34] R. Strydom, S. Thurrowgood, and M. Srinivasan, Visual Odometry: Autonomous UAV Navigation using Optic Flow and Stereo, in Australasian Conf. on Robotics and Automation (ACRA)(Melbourne), 2014.

[35] J. F. Roger-Verdeguer, M. Mannberg and A. Savvaris, Visual odometry with failure detection for the aegis UAV, IEEE International Conference on Imaging Systems and Techniques (IST), pp.291, 296, 16-17 July 2012.

[36] T. Mouats, N. Aouf, L. Chermak, and M. A. Richardson. Thermal Stereo Odometry for UAVs. Sensors Journal, IEEE, vol. 15, no. 11, pp. 6335-6347, 2015.

[37] M. Warren, P. Corke, and B. Upcroft. Long-range stereo visual odometry for extended altitude flight of unmanned aerial vehicles. The International Journal of Robotics Research, 2015. 